\title{
In vivo visualization of propagating a-synuclein pathologies in mouse and marmoset models by a bimodal imaging probe, C05-05
}

\section{Authors}

Maiko Ono ${ }^{1,6 *}$, Manami Takahashi1,6, Aki Shimozawa², Masayuki Fujinaga1, Wakana Mori ${ }^{1}$, Yuji Nagai ${ }^{1}$, Koki Mimura ${ }^{1}$, Katsushi Kumata ${ }^{1}$, Tatsuya Kikuchi ${ }^{1}$, Masafumi Shimojo', Hiroyuki Takuwa1, Takeharu Minamihisamatsu¹, Shoko Uchida$^{1}$, Sushil K. Mishra ${ }^{3}$, Yoshiki Yamaguchi ${ }^{4}$, Hiroshi Shimizu ${ }^{5}$, Akiyoshi Kakita ${ }^{5}$, Hironobu Endo ${ }^{1}$, Yuhei Takado1, Takafumi Minamimoto', Naruhiko Sahara ${ }^{1}$, Ming-Rong Zhang ${ }^{1}$, Masato Hasegawa², Makoto Higuchi ${ }^{1 *}$

\section{Affiliations}

${ }^{1}$ Quantum Life and Medical Science Directorate, National Institutes for Quantum and Radiological Science and Technology, Chiba 263-8555, Japan.

2 Department of Dementia and Higher Brain Function, Tokyo Metropolitan Institute of Medical Science, Tokyo 156-8506, Japan.

3 Department of BioMolecular Sciences, The University of Mississippi, Mississippi 38677, United States.

4 Division of Structural Glycobiology, Institute of Molecular Biomembrane and Glycobiology, Tohoku Medical and Pharmaceutical University, Miyagi 981-8558, Japan.

${ }^{5}$ Department of Pathology, Brain Research Institute, Niigata University, Niigata 951-8585, Japan.

6 These authors contributed equally to this work

*Correspondence: ono.maiko@qst.go.jp, higuchi.makoto@qst.go.jp 


\section{Abstract}

Deposition of intracellular $\alpha$-synuclein fibrils is implicated in neurodegenerative parkinsonian disorders, while high-contrast in vivo detection of a-synuclein depositions has been unsuccessful in animal models and humans. Here, we have developed a bimodal imaging probe, C05-05, for visualizing a-synuclein inclusions in the brains of living animals modeling a-synuclein propagation. In vivo optical and PET imaging of a mouse model demonstrated visualization of $\alpha$ synuclein aggregates by $\mathrm{C} 05-05$, revealing a dynamic propagation of fibrillogenesis along neural pathways followed by disruptions of these structures. Moreover, longitudinal ${ }^{18} \mathrm{~F}-\mathrm{C} 05-05-\mathrm{PET}$ of a marmoset model captured widespread dissemination of fibrillary pathologies accompanied by neurodegeneration detected by dopamine transporter PET. In addition, in vitro assays demonstrated the high-affinity binding of ${ }^{18} \mathrm{~F}-\mathrm{C} 05-05$ to a-synuclein versus other protein pathologies in human brain tissues. Collectively, we propose a new imaging technology enabling etiological and therapeutic assessments of a-synuclein pathogenesis at non-clinical levels, highlighting the applicability of C05-05 to clinical PET.

\section{Introduction}

Parkinson's disease (PD) and dementia with Lewy bodies (DLB) are neurodegenerative diseases of high prevalence, and they are pathologically characterized by the appearance of Lewy bodies and Lewy neurites, which are mainly composed of aggregated $\alpha$-synuclein (1-3). Abnormal $\alpha$-synuclein is also a major component of glial cytoplasmic inclusions (GCls), which are a pathological feature of multiple system atrophy (MSA), a neurodegenerative disease presenting with movement and autonomic disorders (4). In these disorders, referred to as a-synucleinopathies, ultrastructures of a-synuclein filaments containing $\beta$-pleated sheets (5) may display diversity in disease-specific and individually variable manners as revealed by the latest cryo-electron microscopic analysis (6). Previous studies experimentally demonstrated that $\alpha-$ synuclein fibrils acted as templates for the conversion of normal a-synuclein molecules into misfolded species, leading to the prion-like propagation of the $\alpha$ synuclein fibrillogenesis throughout the brain via neural circuits $(7,8)$.

Formation of intracellular $\alpha$-synuclein fibrils is mechanistically linked to neurodegenerative processes, and the spread of a-synuclein inclusions in the 
brain is supposed to be the neuropathological basis of disease progression (911 ), supporting the significance of the $\alpha$-synuclein assembly as a diagnostic and staging biomarker and a therapeutic target. Meanwhile, the diagnosis of PD, DLB, and MSA can only be confirmed by examining the presence of $\alpha$-synuclein aggregates in the autopsied brains, and has been challenging in living subjects. Furthermore, disease-modifying therapeutic approaches to the pathogenetic pathways of $\alpha$-synucleinopathies have been impeded by the lack of antemortem neuropathological investigations of the target protein lesions. Accordingly, imaging techniques capable of detecting a-synuclein aggregates with high sensitivity in the living human brain would provide definitive information on the disease diagnosis at an early stage, and could be of great utility for the evaluation of efficacies yielded by candidate drugs targeting $\alpha$-synuclein pathologies at nonclinical and subsequently clinical levels.

Molecular imaging modalities, as exemplified by positron emission tomography (PET), have enabled visualization of amyloid $\beta$ (12) and tau (13-18) deposits in the brain of living patients with Alzheimer's disease (AD) and related disorders along with mouse models of these illnesses. A significant subset of the PET probes for these proteinopathies is a self-fluorescent $\beta$-sheet ligand and is applicable to intravital two-photon laser fluorescence microscopy of the animal models. Notably, the validity of the macroscopic PET technologies for capturing tau deposits has been proven by two-photon optical imaging of the tau transgenics at a microscopic level, demonstrating a rapid access of the probes to intraneuronal tau aggregates through the blood-brain barrier and neuronal plasma membrane in our previous works $(13,18)$. Hence, there has been growing expectation that small-molecule ligands for $\beta$-sheet structures would also serve as PET and optical probes for multi-scale assessments of intracellular $\alpha$ synuclein fibrils $(19,20)$. However, in vivo visualization of $\alpha$-synuclein aggregates with high contrast has not been successful in the non-clinical and clinical settings. ${ }^{11} \mathrm{C}-\mathrm{BF}-227$, a PET ligand developed to detect amyloid $\beta$ plaques (21), has been reported to bind to $\alpha$-synuclein lesions in the brains of MSA patients in a PET study (22), but in vitro autoradiography of postmortem MSA brain sections in a more recent study did not support significant binding of ${ }^{11} \mathrm{C}-\mathrm{BF}-227$ to $\mathrm{GCls}$ at concentrations typically achieved in PET experiments (23). The tau PET ligand, ${ }^{11} \mathrm{C}-\mathrm{PBB} 3$ was also documented to react with a-synuclein lesions, including Lewy bodies, Lewy neurites, and GCls, while it has been indicated that its binding affinity for $\alpha$-synuclein pathologies is not sufficient for sensitive PET detection of 
these lesions in living individuals $(24,25)$. Indeed, PBB3 shows high affinity and selectivity for the $\beta$-sheet structure of tau filaments, which is assumed to be ultrastructurally distinct from that of a-synuclein assemblies (6, 26-29). In the meantime, the modest binding of PBB3 with a-synuclein inclusions implies its utility as a starting compound for the development of novel derivatives with more appropriate binding properties for in vivo imaging of a-synucleinopathies.

In our screening by in vitro evaluation, we found that derivatives of PBB3 with (E)-hex-2-en-4-yne linker, termed C05 series compounds, exhibited binding to $\alpha$ synuclein pathologies with high reactivity and selectivity compared to PBB3 and BF-227. The in vitro characteristics of a chemical in this class, C05-01, were further analyzed with a tissue microarray in our latest work (30). In the present study, detailed non-clinical evaluations have revealed that another $\mathrm{C05}$ compound, C05-05, has more suitable properties than C05-01 for detection of $\alpha$ synuclein inclusions in murine and non-human primate models of propagating $\alpha$ synuclein pathologies bimodally by in vivo optical and PET imaging from singlecell to brain-wide scales. Furthermore, the high binding affinity of C05-05 for $\alpha$ synuclein inclusions in brain tissues derived from PD, DLB, and MSA cases has supported the applicability of this probe to clinical PET in humans.

\section{Results}

\section{C05 series compounds bind to $\alpha$-synuclein inclusions in vitro}

The tau PET ligand, PBB3, was found to have a moderate affinity for $\alpha$ synuclein pathologies (24), which did not support the suitability of this compound for in vivo imaging of a-synucleinopathies. To develop novel ligands with higher reactivity with $\alpha$-synuclein fibrils than PBB3 and BF-227, we screened PBB3 derivatives by fluorescence staining, in view of the fact that most of these chemicals are self-fluorescent $(13,26)$. We then identified $\mathrm{C} 05$ series compounds, which possess an $(E)$-hex-2-en-4-yne linker in the backbone structure, as candidates for $\alpha$-synuclein imaging agents (Fig. 1a). Binding of C05-05, one of the C05 series compounds, to a-synuclein filaments was also supported by a molecular docking simulation based on ultrastructures of $\alpha$-synuclein assemblies determined by cryo-EM (Supplementary Fig. 1). Double fluorescence staining of DLB brain slices with ligands and antibody against phosphorylated a-synuclein (pS129) demonstrated that C05-01, C05-03, and C05-05 strongly labeled Lewy bodies and Lewy neurites, whereas PBB3 and BF-227 yielded moderate and 
weak fluorescence signals, respectively, on these lesions (Fig. 1b). To compare the binding selectivity for $\alpha$-synuclein versus $A \beta$ and tau pathologies between ligands, fluorescence staining of Lewy bodies and Lewy neurites in DLB amygdala and $A \beta$ plaques and tau tangles in the $A D$ middle frontal gyrus with the C05 series and reference compounds were quantified with a uniform imaging condition (Fig. 1c and Supplementary Fig. 2). The background-corrected fluorescence intensity indicated that the signals attributed to C05-01, C05-03, and C05-05 bound to $\alpha$-synuclein pathologies were significantly higher than those of $A \beta$ and tau (Fig. 1d), suggesting that the selectivity of C05 series compounds for $\alpha$-synuclein versus $A \beta$ and tau aggregates in the human brains. In contrast, the fluorescence signals originating from tau-bound PBB3 were significantly higher than those of this compound attached to $\alpha$-synuclein and $A \beta$ deposits, and the fluorescence signals attributed to $A \beta$-bound BF-227 were significantly higher than those of this compound attached to a-synuclein and tau deposits.

\section{C05-05 enables in vivo optical visualization of $\alpha$-synuclein inclusions in the brains of mice modeling propagating $\alpha$-synucleinopathy}

For assessing in vivo detectability of intracellular a-synuclein deposits by $\mathrm{C05}$ series compounds at an individual cell level, we utilized a mouse model of propagating a-synuclein fibrillogenesis induced by inoculation of recombinant mouse $\alpha$-synuclein fibrils into the brain parenchyma of a wild-type mouse ( $\alpha$-Syn mouse) (8). In the a-Syn mouse, aggregates of phosphorylated endogenous $\alpha$ synuclein molecules resembling Lewy bodies and Lewy neurites emerged bilaterally in extensive brain regions, including the striatum, cortex, amygdala and substantia nigra, from 2 weeks after unilateral inoculation of a-synuclein fibrils into the striatum (Supplementary Fig. 3). Double-staining of brain slices with fluorescent compounds and pS129 demonstrated that C05-01, C05-03, and C0505 intensely labeled pS129-positive phosphorylated a-synuclein inclusions similar to Lewy bodies and Lewy neurites in the neocortex of $\alpha$-Syn mice, while PBB3 and BF-227 bound modestly with these deposits (Fig. 1e). We selected C05-01 and C05-05 for the following characterizations in consideration of their suitability for ${ }^{18} \mathrm{~F}$ radiolabeling towards wider availability.

To assess the time course of in vivo labeling of intraneuronal $\alpha$-synuclein inclusions with C05-01 and C05-05 compared with PBB3, we conducted intravital two-photon laser fluorescence microscopy of the somatosensory cortex of an $\alpha$ Syn mouse through a cranial window. Detection of C05-05, C05-01, and PBB3 
signals in the same field of view of a single individual animal indicated rapid entry of C05-05 into the brain after intraperitoneal administration, reaching a-synuclein inclusions within $5 \mathrm{~min}$, and the binding continued for $90 \mathrm{~min}$ (Fig. 2). Unlike C0505 , no noticeable increases in fluorescence signals were produced in neurons by intraperitoneally injected C05-01 and PBB3. We did not employ BF-227 as a reference compound in these assays, as its fluorescence wavelength did not fit intravital observations. Ex vivo examination of frozen brain sections from a-Syn mouse following intravital two-photon microscopy further proves the binding of intraperitoneally administered $\mathrm{C05-05}$ to a-synuclein inclusions abundantly present in the somatosensory cortex of this mouse (Supplementary Fig. 4).

Ex vivo examination of brain tissues collected from an a-Syn mouse at two hours after intraperitoneal BF-227 injection demonstrated no apparent interaction of this compound with a-synuclein inclusions stained with pS129 (Fig. 3a and b). In contrast, brain samples collected from the a-Syn mouse at 90 min after intraperitoneal C05-05 injection contained numerous fibrillary inclusions labeled with the injected compound in broad areas of the brain, including the striatum, neocortex and amygdala, and these aggregates were subsequently stained with pS129, proving the entry of C05-05 into the brain followed by attachment of this ligand to intraneuronal a-synuclein assemblies (Fig. $3 c$ and d). Ex vivo examination of brain tissues collected from a wild-type control mouse injected with saline into the striatum showed no apparent distribution of intraperitoneally administered BF-227 and C05-05 in the cerebral parenchyma (Supplementary Fig. 5). These in vivo and ex vivo data collectively demonstrate the capability of C05-05 for high-contrast optical visualization of a-synuclein inclusions in a living a-synucleinopathy mouse model.

\section{In vivo microscopy with C05-05 allows tracking of pathological a-synuclein propagation through neural processes in the brain of a mouse model}

To assess the dissemination of fibrillary $\alpha$-synuclein pathologies via neuronal processes and cell bodies and consequent disruptions of these cellular structures on a longitudinal basis, we performed biweekly intravital two-photon imaging of a-synuclein inclusions in the brain of an a-Syn mouse inoculated with a-synuclein fibrils into the unilateral striatum. Prior to in vivo assessments, histopathological examinations of brain sections collected from a-Syn mice at several time points after the inoculation suggested the appearance of endogenous a-synuclein aggregates in neurites at two weeks, transient increase of inclusions in neurites 
and soma at four weeks, and subsequent decline of neuritic inclusions and maturation of somatic aggregates in the somatosensory cortex ipsilateral to the inoculation (Fig. 4a).

Correspondingly, in vivo longitudinal microscopic imaging in a-Syn mice by two-photon laser scanning with $\mathrm{C05-05}$ demonstrated the spatiotemporal changes of $\alpha$-synuclein inclusions within an individual. Neuritic a-synuclein accumulations labeled with C05-05 appeared abundantly in the somatosensory cortex of the inoculated hemisphere at four weeks and then decreased at six weeks (Fig. 4b, white arrowheads). Moreover, the formation and growth of somatic $\alpha$-synuclein inclusions labeled with C05-05 were observed at 8 and 12 weeks after inoculation (Fig. 4b, yellow arrowheads). High-magnification images clearly visualized the intraneuronal expansion of pathological $\alpha$-synuclein aggregates from neuritic to somatic compartments in a week (Fig. 4c). Moreover, time-course assays provided more compelling evidence for the disappearance of neuritic (Fig. 4d, top) and somatic (Fig. 4d, bottom) a-synuclein inclusions in two weeks and demonstrated loss of mCherry-expressing neurons bearing C05-05positive a-synuclein fibrils (Fig. 4e). These in vivo data along the course after the inoculation of $\alpha$-synuclein fibrils clarified stretching of $\alpha$-synuclein depositions inside a single neuron, serially inducing neuritic and somatic fibril formations and subsequent breakdowns of neuronal structures, which is suggestive of $\alpha$ synuclein-provoked neurotoxic insults. Our findings also demonstrated the utility of C05-05 as an optical probe for a dynamic pursuit of the neurodegenerative $\alpha-$ synuclein pathogenesis at a single-cell level.

\section{PET imaging with ${ }^{18} \mathrm{~F}-\mathrm{C} 05-05$ visualizes depositions and propagations of pathological $\alpha$-synuclein species in the brains of living model animals}

To assess the in vivo performance of ${ }^{18} \mathrm{~F}$-labeled $\mathrm{C} 05-05\left({ }^{18} \mathrm{~F}-\mathrm{C} 05-05\right.$, Supplementary Fig. 6) as a PET ligand, we performed PET scans with ${ }^{18} \mathrm{~F}-\mathrm{C} 05-$ 05 for $\alpha$-Syn mice at six months after injection of $\alpha$-synuclein fibrils or saline into the bilateral striata, followed by ex vivo autoradiography and histopathological examinations. As depicted in Fig. 5a, the retention of radioligand was overtly increased in the bilateral striatal and cortical areas of an a-Syn mouse, in sharp contrast to the low radioactivity signals sustained in these brain regions of a control mouse. ${ }^{18} \mathrm{~F}-\mathrm{C} 05-05$ rapidly entered the brain after intravenous administration, and peak radioactivity uptakes estimated as standardized uptake values (SUVs) were 1.19 and 1.11 in the striatum and cortex, respectively (Fig. 
$5 b$, top). This was followed by a washout of radioactivity from the brain of control mice, whereas the clearance was retarded in the striatum and cortex of a-Syn mice, reflecting radioligand binding to a-synuclein deposits. In the cerebellum lacking a-synuclein pathologies, there was no clear difference in the retention of radioligand between $\alpha-S y n$ and control mice (Fig. 5a, bottom, and 5b, top), justifying the use of the cerebellum as a reference tissue for quantification of the radioligand binding. The target-to-reference ratios of the radioactivity, which is denoted as standardized uptake value ratio (SUVR), at each time point and average SUVR at 90-120 min after intravenous administration of ${ }^{18} \mathrm{~F}-\mathrm{C} 05-05$ were increased in the striatum and cortex of a-Syn mice compared to those of control mice (Fig. 5b, bottom).

Ex vivo autoradiography of brain tissues collected from a-Syn and control mice used for PET scan at 90 min after intravenous ${ }^{18} \mathrm{~F}-\mathrm{C} 05-05$ injection demonstrated accumulation of the radioligand in the striatum, cortex and amygdala of the $\alpha-S y n$ mouse harboring abundant neuronal a-synuclein inclusions (Fig. 5c and d). Conversely, there was no noticeable increase of radioligand retentions in these brain regions of the control mouse. In addition, the radioligand accumulation was minimal in the cerebellum of the a-Syn and control mice, the area devoid of $\alpha$ synuclein deposits, while non-specific radioligand accumulations in several white matter regions, including the corpus callosum and fimbria of the hippocampus, was observed in both of these mice.

Since the small brain volumes of mice impeded clear separations between striatal and neocortical radioactivity signals as assessed by $\mathrm{PET}$, the in vivo traceability of the inter-regional $\alpha$-synuclein dissemination with the use of ${ }^{18} \mathrm{~F}$ C05-05 remained rather inconclusive. We accordingly employed a non-human primate model of propagating a-synuclein pathologies by inoculating recombinant marmoset $\alpha$-synuclein fibrils into the brain parenchyma ( $\alpha$-Syn marmoset). Our previous work documented that marmosets inoculated with murine a-synuclein fibrils displayed conversion of endogenous a-synuclein molecules into fibrillary aggregates, resulting in abundant accumulations of phosphorylated $\alpha$-synuclein inclusions resembling Lewy bodies and Lewy neurites in the inoculation sites and subsequently remote brain areas through the neural network (31). It was noteworthy that the retrograde propagation of pathological $\alpha$-synuclein species from the caudate nucleus and putamen to substantia nigra through the nigrostriatal dopaminergic pathway was prominent at 3 months after inoculation (31). Similar to this model, an a-Syn marmoset receiving marmoset $\alpha$-synuclein 
fibrils exhibited enhanced retention of ${ }^{18} \mathrm{~F}-\mathrm{C} 05-05$ in a sub-portion of the caudate nucleus containing the injection site at 1 month after inoculation, which spread extensively in the caudate nucleus, putamen, and substantia nigra of the ipsilateral hemisphere and to a lesser extent in the contralateral left hemisphere at three months (Fig. 6a). We also performed PET imaging of dopamine transporters with a specific radioligand, ${ }^{11} \mathrm{C}-\mathrm{PE} 2 \mathrm{I}$, which has proven useful for detecting degenerations of dopaminergic neurons in PD and its models $(32,33)$, in the $\alpha$-Syn marmoset before (Pre) and 3 months after inoculation. Parametric images of ${ }^{11} \mathrm{C}-\mathrm{PE} 2 \mathrm{I}$ binding potential (BPND) demonstrated a decrease of dopamine transporters in the caudate nucleus, putamen, and substantia nigra of the inoculated hemisphere compared to the contralateral hemisphere, in agreement with the distribution of augmented ${ }^{18} \mathrm{~F}-\mathrm{C} 05-05$ retentions and pathological a-synuclein depositions (Fig. 6b).

The brain of this animal was sampled at four months after inoculation, and immunohistochemical analyses of the brain slices demonstrated the distribution of pS129-stained a-synuclein inclusions in agreement with in vivo PET findings with ${ }^{18} \mathrm{~F}-\mathrm{C} 05-05$ at three months (Fig. 6c). Double-staining with C05-05 and pS129 confirmed dense accumulations of a-synuclein aggregates in neuronal processes and somas recapitulating PD and DLB pathologies in the caudate nucleus, putamen, and substantia nigra of the inoculated hemisphere (Fig. 6d). The corresponding brain areas of the contralateral hemisphere contained less abundant $\alpha$-synuclein inclusions in neurites and neuronal somas. These $\alpha-$ synuclein pathologies were fluorescently labeled with C05-05, suggesting that the increased retention of radioligand in ${ }^{18} \mathrm{~F}-\mathrm{C} 05-05-\mathrm{PET}$ stemmed from its in vivo interaction with a-synuclein inclusions (Fig. 6a and d). Meanwhile, non-specific accumulations of radioligand in bilateral white matter regions flanking the putamen were noted in the pre-inoculation ${ }^{18} \mathrm{~F}-\mathrm{C} 05-05-P E T$ (Fig. 6a, top), and the absence of $\alpha$-synuclein deposits in these areas was ensured by histochemical and immunohistochemical assays.

These in vivo data provide the first PET demonstration of time-course imaging of pathological $\alpha$-synuclein deposits in living animal models along the course of spatially expanding fibrillogenesis accompanied by the degeneration of neural circuits involved as dissemination pathways.

\section{${ }^{18} \mathrm{~F}-\mathrm{C05}-05$ displays high-affinity binding to $\alpha$-synuclein pathologies in DLB, PD and MSA brain tissues}


To assess binding of ${ }^{18} \mathrm{~F}-\mathrm{C} 05-05$ to human a-synuclein pathologies at a low concentration (10 $\mathrm{nM})$, we performed in vitro autoradiography of basal ganglia from MSA and Parkinson's disease with dementia (PDD) cases and amygdala from DLB case (Fig. 7a). The total binding of ${ }^{18} \mathrm{~F}-\mathrm{C} 05-05$ was markedly abolished by excessive non-radiolabeled $\mathrm{C05}-05$, indicating the saturability of the radioligand binding. The MSA cases showed specific binding of ${ }^{18} \mathrm{~F}-\mathrm{C} 05-05$ in association with the local $\alpha$-synuclein burden (Fig. 7a and b). A case with mild pathology (MSA-1) had no binding of ${ }^{18} \mathrm{~F}-\mathrm{C} 05-05$ to the striatopallidal fibers. MSA-2, which was burdened with moderate a-synuclein deposits, showed weak ${ }^{18} \mathrm{~F}-\mathrm{C} 05-05$ radioactivity signals in striatopallidal fibers containing numerous $\mathrm{GCls}$. MSA-3, which is a case with severe a-synuclein pathologies, exhibited intense radioligand binding to the striatopallidal fibers harboring densely packed $\mathrm{GCls}$. The DLB and PDD cases also showed specific binding of ${ }^{18} \mathrm{~F}-\mathrm{C} 05-05$ in line with the distribution of a-synuclein pathology in the amygdala and substantia nigra, respectively.

We then conducted triple staining of the sections used for autoradiography with C05-05 and antibodies against a-synuclein (LB509) and antibody against phosphorylated tau (pS199/202). The fluorescence labeling with C05-05 and LB509 was noted on GCls in the MSA striatopallidal fibers, and Lewy bodies and Lewy neurites in the DLB amygdala and PDD substantia nigra, and these areas were devoid of pS199/202-immunoreactive phosphorylated tau pathologies (Fig. 7b).

We also quantified the affinity of ${ }^{18} \mathrm{~F}-\mathrm{C} 05-05$ for a-synuclein aggregates in homogenized DLB amygdala tissues in comparison to AD frontal cortical tissues. Radioligand binding in these tissues was homologously blocked by nonradiolabeled C05-05 in a concentration-dependent fashion (Fig. 7c), indicating binding saturability. ${ }^{18} \mathrm{~F}-\mathrm{C} 05-05$ displayed high-affinity binding in DLB homogenates with the concentration inducing 50\% homologous inhibition (IC50) of $1.5 \mathrm{nM}$ (Fig. 7f). This radioligand was not highly binding with $A \beta$ and tau aggregates in AD tissues relative to DLB a-synuclein deposits, with IC50 of 12.9 nM. Unlike ${ }^{18} \mathrm{~F}-\mathrm{C} 05-05$, tau PET tracers, ${ }^{11} \mathrm{C}-\mathrm{PBB} 3$ and ${ }^{18} \mathrm{~F}-\mathrm{PM}-\mathrm{PBB} 3$, displayed relatively low affinities for $\alpha$-synuclein deposits in DLB homogenates with IC50 values of $58.8 \mathrm{nM}$ and $26.5 \mathrm{nM}$, respectively, while these radioligands more tightly bound to AD-type protein fibrils than a-synuclein aggregates, with IC50 values of $8.6 \mathrm{nM}$ and $8.0 \mathrm{nM}$, respectively (Fig. 7d-f). The molecular docking analyses based on the cryo-EM structure also suggested that $\mathrm{C} 05-05$ binds to the potential 
binding sites in MSA-type a-synuclein filaments with a stronger affinity than PBB3 and PM-PBB3 (Supplementary Fig. 1). These results of in vitro autoradiographic and radioligand binding assays highlight the reactivity of ${ }^{18} \mathrm{~F}-\mathrm{C} 05-05$ with human a-synuclein pathologies with much higher affinity than existing PET tracer for nona-synuclein aggregates, supporting the potential of this novel radioligand for visualizing hallmark lesions in living patients with $\alpha$-synucleinopathies.

\section{Discussion}

The current work has offered a powerful imaging tool to pursue the molecular and cellular mechanisms of the neurodegenerative $\alpha$-synucleinopathies in basic research on animal models, and this technology might be translatable to clinical PET assessments of PD and associated disorders. The imaging methodology with ${ }^{18} \mathrm{~F}-\mathrm{C} 05-05$ potentially meets the needs for the early diagnosis and differentiation of neurocognitive and movement disorders by targeting neurotoxic fibrillary species of a-synuclein molecules, along with the discovery and development of anti-a-synuclein therapeutics as disease-modifying treatments $(34,35)$. Our bimodal in vivo optical and PET assays have allowed longitudinal tracking of a-synuclein propagations through neural pathways from subcellular to brain-wide scales, facilitating the non-clinical evaluation of efficacies exerted by a candidate drug counteracting the etiological processes of $\alpha$-synucleinopathies.

It is noteworthy that the substitution of the $(2 E, 4 E)$-hexa-2, 4-dien linker in the chemical structure of a tau imaging agent, PBB3, with $(E)$-hex-2-en-4-yne resulted in a profound increase of the ligand binding to $\alpha$-synuclein versus tau and $A \beta$ fibrils, leading to the generation of $\mathrm{C} 05$ series compounds. The molecular docking analyses based on the cryo-EM structure suggested that PBB3 and C0505 bind to AD-type tau filaments and MSA-type a-synuclein filaments, respectively, in a direction perpendicular to the fibril axis (Supplementary Fig. 1) (28). The linker substitution could produce differences in the backbone twist angle between PBB3 and C05 series compounds at a minimum energy state, which may affect the fitness of the chemical for the binding surface on the filament. Our molecular docking analysis suggested that C05-05 binds to the potential binding sites in MSA-type a-synuclein filaments with a stronger affinity than PBB3 and PM-PBB3. In fact, IC50 of C05-05 for the homologous binding blockade in homogenized DLB amygdala tissues was approximately 40- and 18-fold smaller than those of PBB3 and PM-PBB3, respectively, implying the critical role of the 
linker angle in the ligand affinity for pathological fibrils. It is yet to be clarified whether C05 series compounds exhibit differential reactivity with PD, DLB, and MSA a-synuclein fibrils, although autoradiographic labeling of pathological inclusions in those illnesses was demonstrated with ${ }^{18} \mathrm{~F}-\mathrm{C} 05-05$ in the present assay. It has been reported that the ultrastructure of tau fibrils shows diversity among AD, Pick's disease, and corticobasal degeneration $(27,29,36)$, and this variation could underlie distinct binding of tau PET probes to AD versus non-AD tau pathologies $(18,26)$. On the analogy of these insights, it will be required to assess the in vitro interaction of $\mathrm{C05-05}$ and related chemicals with aggregates in various $\alpha$-synucleinopathies, which will provide useful information for predicting the in vivo performance of the probes in clinical PET imaging of cases with these disorders.

Intravital two-photon laser microscopy with $\mathrm{C05-05}$ has enabled longitudinal imaging of the $\alpha$-synuclein fibrillogenesis at a subcellular scale in the brain of a living a-synucleinopathy mouse model for the first time, visualizing the dynamic processes in the formation of a-synuclein lesions, including a spatiotemporal connection between the developments of Lewy neurite-like neuritic aggregates and Lewy body-like somatic inclusion in a single neuron, as well as the disappearance of these fibrillar deposits. While we found evidence that the vanishment of $\alpha$-synuclein fibrils reflects the loss of neurons loaded with inclusions, the dynamic appearance and disappearance of the aggregates may also unfold as a consequence of continuous translocations of $\alpha$-synuclein assemblies through neuritic processes. The mechanisms linking the accumulations of $\alpha$-synuclein fibrils and loss of neurons or their substructures remain elusive, and cell-autonomous (37) and non-cell-autonomous (38) death of neurons could be provoked in the pathogenetic pathway. Such etiological cellular events will be microscopically examined by monitoring interactions between glial cells expressing fluorescent proteins and neurons bearing C05-05positive $\alpha$-synuclein deposits. Meanwhile, the transient accumulation of $\alpha$ synuclein aggregates in neuronal compartments may indicate the transport of these pathological components along neurites, but it is also presumable that a significant portion of the fibrils could be degraded by autophagic and other related processes. In addition, the stretching of a-synuclein depositions inside neurites towards the cell body might be caused by a domino-like conversion of endogenous a-synuclein molecules to misfolded forms prone to the selfaggregation, whereas dislocation of native $\alpha$-synuclein proteins from presynaptic 
to neuritic and somatic compartments should be necessary for this involvement. The localization of endogenous a-synuclein molecules and their engagement in the fibril formation would be investigated in detail by expressing fused $\alpha$-synuclein and fluorescent proteins (39) in neurons of a-Syn mice, which could be used for intravital microscopic assays with C05-05.

Since our longitudinal PET scans with ${ }^{18} \mathrm{~F}-\mathrm{C} 05-05$ have successfully captured the dissemination of $\alpha$-synuclein pathologies in an $\alpha$-Syn marmoset along the course following the fibril inoculation, this imaging technology will pave the way to the neuroimaging-based evaluations of the disease severity and progression in a-synucleinopathy patients. The topology of a-synuclein pathology and its chronological change are known to be closely correlated with the symptomatic phenotypes (9-11), indicating the local neurotoxicity of aggregated a-synuclein molecules. In the marmoset model of $\alpha$-synuclein propagation, intensification and expansion of $\alpha$-synuclein depositions visualized by ${ }^{18} \mathrm{~F}-\mathrm{C} 05-05$ were in association with declines of the nigral dopaminergic neurons and their striatal terminals as assessed by PET imaging of dopamine transporters, in resemblance to the dopaminergic deficits in PD. This observation also implies that ${ }^{18} \mathrm{~F}-\mathrm{C} 05-05$ could illuminate $\alpha$-synuclein species critically involved in functional and structural disruptions of neurons. Previous studies suggested linkage of misfolded $\alpha$ synuclein proteins with synaptic dysfunctions, such as a decrease of soluble $\mathrm{N}$ ethylmaleimide-sensitive factor attachment protein receptor (SNARE) complex assembly and synaptic vesicle motility $(40,41)$. Influences of C05-05-detectable a-synuclein accumulations on the functionality of individual neurons will be examined by conducting intravital two-photon microscopy of a-Syn mice expressing calcium sensor proteins, and this assay system would be utilized for obtaining pathological and functional outcome measures in the non-clinical evaluation of a candidate therapeutic agent.

The total amount of abnormal $\alpha$-synuclein proteins was reported to be approximately 50 - $200 \mathrm{nM}$ in the brainstem and subcortical regions of advanced DLB and MSA cases, which was more than 10-fold smaller than the amount of $A \beta$ peptide deposited in the brain of $A D$ patients (42). This finding raises a concern on the visibility of $\alpha$-synuclein pathologies by PET in a clinical setting. The detection of $\alpha$-synuclein pathologies in the brain of murine and non-human primate models was permitted by appropriate pharmacokinetic and pharmacodynamic characteristics of this probe in living animals. The high reactivity of $\mathrm{C} 05$ series compounds with $\alpha$-synuclein inclusions relative to $A \beta$ and 
tau deposits was demonstrated by in vitro fluorescence staining and binding assays, and IC50 of ${ }^{18} \mathrm{~F}-\mathrm{C} 05-05$ for the homologous blockade of its binding to $\alpha$ synuclein aggregates in DLB tissue was $5-6$ times lower than those of ${ }^{11} \mathrm{C}-\mathrm{PBB} 3$ and ${ }^{18} \mathrm{~F}-\mathrm{PM}-\mathrm{PBB} 3$ for the self-blockade of their binding to tau fibrils in AD tissue. Accordingly, PET with ${ }^{18} \mathrm{~F}-\mathrm{C} 05-05$ would visualize $\alpha$-synuclein depositions in DLB cases even if the brains of these patients possess radioligand binding sites with 5-fold lower density than AD brains. In contrast, IC50 values of ${ }^{11}$ C-PBB3 and ${ }^{18} \mathrm{~F}-\mathrm{PM}-\mathrm{PBB} 3$ for the self-blockade of their binding in DLB brain homogenates were much higher than that of ${ }^{18} \mathrm{~F}-\mathrm{C} 05-05$, leading to the notion that these tau ligands are unlikely to detect $\alpha$-synuclein fibrils in living individuals with sufficient sensitivity. Furthermore, our histological data illustrated that a-synuclein pathologies in the midbrain of an $\alpha$-Syn marmoset were less abundant than pathological deposits in the DLB amygdala, but these non-human primate deposits were detectable by ${ }^{18} \mathrm{~F}-\mathrm{C} 05-05-\mathrm{PET}$. These facts could bring an implication on the usability of ${ }^{18} \mathrm{~F}-\mathrm{C} 05-05$ for imaging of core pathologies in $\alpha$ synucleinopathy cases.

In addition to the reactivity with the target lesion, the entry of the compound into the brain is a key factor for yielding a high signal-to-noise ratio in PET neuroimaging. Among C05 series compounds, C05-01 and C05-05 were fluorinated chemicals with desirable in vitro binding properties (Fig. 1d) (30). However, visualization of $\alpha$-synuclein aggregates by intravital two-photon microscopic imaging of model mice was unsuccessful with c05-01. It is conceivable that the hydroxy moiety of C05-01 could be promptly conjugated with sulfate after systemic administration in a mode similar to PBB3 (43), and such metabolic modification was circumvented by the replacement of this structural group with a fluoroisopropanol moiety in PM-PBB3 (18) and C05-05 (Supplementary Fig. 7), increasing the amount of the intact compound entering the brain. In fact, the peak radioactivity uptake of ${ }^{18} \mathrm{~F}-\mathrm{C} 05-05$ in the frontal cortex (SUV, 1.11; Fig. 5b) was approximately 2-fold higher than that of ${ }^{18} \mathrm{~F}-\mathrm{C} 05-01$ (SUV, 0.58; Supplementary Fig. 8) in PET imaging of wild-type control mice. In addition, ${ }^{18} \mathrm{~F}-\mathrm{C} 05-05$ has been confirmed to be decomposed to a hydrophilic radiometabolite in plasma, suggesting lower entry of the radiometabolite than unmetabolized compound into the brain (Supplementary Fig. 7).

Despite promising features of ${ }^{18} \mathrm{~F}-\mathrm{C} 05-05-\mathrm{PET}$ as a neuroimaging technique translatable from animal models to humans, several technical issues are yet to be addressed. In $\alpha$-Syn mice, PET with ${ }^{18} \mathrm{~F}-\mathrm{C} 05-05$ visualized abundant $\alpha-$ 
synuclein accumulation in the striatum and cortex, but failed to detect abundant a-synuclein accumulation in amygdala (Supplementary Fig. 9). Ex vivo examination of brain tissues collected from the model mice confirmed the binding of ${ }^{18} \mathrm{~F}$-C05-05 systemically administered to $\alpha$-synuclein inclusions deposited in this area. Therefore, the incapability of ${ }^{18} \mathrm{~F}-\mathrm{C} 05-05-\mathrm{PET}$ for capturing target pathologies in the amygdala is attributable to the limited spatial resolution of the imaging device and consequent partial volume effects, which might preclude in vivo neuropathological assessments in a relatively small anatomical structure. A possible solution to this issue could be the use of a non-human primate model with a larger brain volume, but it should be taken into account that non-specific accumulation of radioligand in white matter regions was observed by ${ }^{18} \mathrm{~F}-\mathrm{C} 05-05-$ PET imaging of a marmoset model even before inoculation of $\alpha$-synuclein fibrils. Notwithstanding these pathology-unrelated signals, ${ }^{18} \mathrm{~F}-\mathrm{C} 05-05-\mathrm{PET}$ could detect increased radioligand retention associated with $\alpha$-synuclein inclusions in white matter structures. In the clinical application of ${ }^{18} \mathrm{~F}-\mathrm{C} 05-05$ to humans, non-specific radioactivity signals in the white matter might impede imaging of a-synuclein lesions, particularly in the MSA brains, since GCls accumulate in white matter areas such as deep cerebellar structures (44). Moreover, several tau PET ligands are known to show off-target binding to monoamine oxidases A and B (45-47). By contrast, previous studies documented that ${ }^{11} \mathrm{C}-\mathrm{PBB} 3$ and its analogs, including ${ }^{18} \mathrm{~F}-\mathrm{PM}-\mathrm{PBB} 3$ and ${ }^{18} \mathrm{~F}-\mathrm{C} 05-01$, did not cross-react with these enzymes $(18,30,48)$, and our in vitro binding assay suggested insensitivity of ${ }^{18} \mathrm{~F}-\mathrm{C} 05-05$ to monoamine oxidases (Supplementary Fig. 10).

In the present study, we granted the highest priority to the sensitive PET detection of $\alpha$-synuclein pathologies with a high-affinity radioligand, as such a goal has been reached in neither animal models nor humans. In the brains of $\alpha-$ synucleinopathy patients, $\alpha$-synuclein lesions are often co-localized with $A \beta$ and tau aggregates. Tau pathologies at Braak stage III or above and A $\beta$ pathologies are observed in more than $50 \%$ and $80 \%$ of $\alpha$-synucleinopathy patients, respectively (49). This fact raises the necessity for the development of a specific ligand for a-synuclein deposits with minimal cross-reactivity with other pathological fibrils. ${ }^{18} \mathrm{~F}-\mathrm{C} 05-05$ displayed more than eight times smaller IC50 (1.5 $\mathrm{nM})$ in DLB homogenates than in AD homogenates (12.9 nM), but its reactivity with tau deposits might not be markedly lower than that of ${ }^{11} \mathrm{C}-\mathrm{PBB} 3$ and ${ }^{18} \mathrm{~F}-\mathrm{PM}$ PBB3. In view of the putative structure-activity relationships indicated in this study, however, we are able to take advantage of $\beta$-sheet ligands with the $(E)$-hex-2-en- 
4-yne linker as potent binders, and structural modifications will be made for enhancing the selectivity of the chemicals by replacing aromatic rings and sidechains. It is also of significance that optical and PET imaging modalities can be utilized for the characterization of new candidate imaging agents.

To conclude, the current neuroimaging platform incorporating C05-05 is implementable for multi-scale analysis of the neurodegenerative $\alpha$-synuclein fibrillogenesis and pharmacological actions of a drug candidate on this etiological process in animal models. Our assays have also provided essential information on the feasibility of ${ }^{18} \mathrm{~F}-\mathrm{C} 05-05$ for the first demonstration of $\alpha$-synuclein PET imaging in humans.

\section{Online methods}

\section{Experimental animals}

All animals studied here were maintained and handled in accordance with the National Research Council's Guide for the Care and Use of Laboratory Animals. Protocols for the present animal experiments were approved by the Animal Ethics Committees of the National Institutes for Quantum and Radiological Science and Technology (approval number: 07-1049-31, 11-1038-11). A total of 35 adult C57BL/6J mice (male, mean age 5.4 months, Japan SLC Inc) were used for the histochemical analysis, ex vivo examination, two-photon microscopy and PET scanning, and one adult marmoset (male, 2 years old, 300-365 g body weights) was used for PET scanning and histochemical analysis in this study. All mice and the marmoset were maintained in a 12 hours' light/dark cycle with ad libitum access to standard diet and water.

\section{Compounds and antibodies}

C05-01 ((E)-2-(4-(2-fluoro-6-(methylamino)pyridine-3-yl)but-1-en-3-yn-1yl)benzo[d]thiazol-6-ol), C05-03 ((E)-2-(4-(6-(methylamino)pyridin-3-yl)but-1-en3-yn-1-yl)benzo[d]thiazol-6-ol), $\quad$ C05-05 $\quad((E)-1-f l u o r o-3-((2-(4-(6-$ (methylamino)pyridine-3-yl)but-1-en-3-yn-1-yl)benzo[d]thiazol-6-yl)oxy)propan2-ol), $\quad$ PBB3 (2-((1E,3E)-4-(6-(methylamino)pyridine-3-yl)buta-1,3dienyl)benzo[d]thiazol-6-ol), desmethyl precursor of ${ }^{11} \mathrm{C}-\mathrm{PBB} 3, \quad$ PM-PBB3 1fluoro-3-((2-((1E,3E)-4-(6-(methylamino)pyridine-3-yl)buta-1,3-dien-1-

yl)benzo[d]thiazol-6-yl)oxy)propan-2-ol, tosylate precursor of ${ }^{18} \mathrm{~F}-\mathrm{PM}-\mathrm{PBB} 3$, and desmethyl precursor of ${ }^{11} \mathrm{C}-\mathrm{PE} 2 \mathrm{I}$ were custom-synthesized (Nard Institute and 
KNC Laboratories). BF-227 (Nard Institute, NP039-0), clorgiline (Sigma-Aldrich, M3778), selegiline (Sigma-Aldrich, NMID822), and sulforhodamine 101 (SigmaAldrich, S7635) are commercially available. Monoclonal antibodies against $\alpha-$ synuclein phosphorylated at Ser 129 (pS129; abcam, ab59264), a-synuclein (LB509; abcam, ab27766), tau phosphorylated at Ser 202 and Thr 205 (AT8; ThermoFisher Scientific, MN1020), and amyloid $\beta$ (6E10; BioLegend, 803004), and polyclonal antibody against tau phosphorylated at Ser 199 and Thr 202 (pS199/202; ThermoFisher Scientific, 44-768G) are commercially available. All experiments with C05-01, C05-03, C05-05, ${ }^{18} \mathrm{~F}-\mathrm{C} 05-05$, PBB3, ${ }^{11} \mathrm{C}-\mathrm{PBB} 3, \mathrm{PM}-$ PBB3, and ${ }^{18}$ F-PM-PBB3 were performed under UV-cut light to avoid photoisomerization of these compounds (18).

\section{Postmortem brain tissues}

Postmortem human brains were obtained from autopsies carried out at the Department of Neuroscience of the Mayo Clinic on patients with DLB and MSA, at the Center for Neurodegenerative Disease Research of the University of Pennsylvania Perelman School of Medicine on patients with $A D$, and at the Department of Pathology of Niigata University on patients with PDD. Tissues for homogenate binding assays were frozen, and tissues for histochemical, immunohistochemical and autoradiographic labeling were frozen or fixed in $10 \%$ neutral buffered formalin followed by embedding in paraffin blocks.

\section{Preparation of recombinant $\alpha$-synuclein and fibrils}

Recombinant mouse and marmoset wild-type a-synuclein and fibrils were prepared as described previously $(8,31,50)$. Briefly, purified a-synuclein (7 -10 $\mathrm{mg} / \mathrm{mL}$ ) was incubated at $37^{\circ} \mathrm{C}$ in a shaking incubator at $200 \mathrm{rpm}$ in $30 \mathrm{mM}$ Tris$\mathrm{HCl}, \mathrm{pH} 7.5$, containing $0.1 \% \mathrm{NaN}_{3}$, for 72 hours. $\alpha$-synuclein fibrils were pelleted by spinning the assembly mixtures at $113,000 \times \mathrm{g}$ for $20 \mathrm{~min}$, resuspended in saline, and sonicated for $3 \mathrm{~min}$ (Biomic 7040 Ultrasonic Processor, Seiko). Protein concentrations were determined by high performance liquid chromatography (HPLC) and adjusted to $4 \mathrm{mg} / \mathrm{mL}$ by dilution with saline.

\section{Virus preparation}

A recombinant adeno associated virus (AAV) was prepared in HEK293T cells by polyethylenimine mediated co-transfection of AAV transfer vector encoding mCherry with rat Synapsin promoter and AAV serotype DJ packaging plasmids, 
pHelper and pRC-DJ (Cell Biolabs Inc.), as described previously (51). 48 hours after transfection, cells were harvested and lysed in $20 \mathrm{mM} \mathrm{HEPES-NaOH}, \mathrm{pH}$ 8.0, $150 \mathrm{mM} \mathrm{NaCl}$ buffer supplemented with $0.5 \%$ sodium deoxycholate and 50 units/mL benzonase nuclease (Sigma-Aldrich). AAV particles were next purified with HiTrap heparin column (GE Healthcare) and virus titer was determined by AAVpro® Titration kit (for Real Time PCR) ver2 (TaKaRa).

\section{Stereotaxic surgery}

For histochemistry, ex vivo examination and in vivo longitudinal imaging by twophoton laser scanning, nine-week-old mice anesthetized with $1.5 \%(\mathrm{v} / \mathrm{v})$ isoflurane were unilaterally injected with $3 \mu \mathrm{L}$ of recombinant mouse $\alpha$-synuclein fibrils or $3 \mu \mathrm{L}$ of saline into striatum (Interaural $3.82 \mathrm{~mm}$, Lateral $2.0 \mathrm{~mm}$, Depth $2.0 \mathrm{~mm}$ ) via glass capillary. For PET study and ex vivo autoradiography, nineweek-old mice anesthetized with $1.5 \%(\mathrm{v} / \mathrm{v})$ isoflurane were bilaterally injected with $3 \mu \mathrm{L}$ of recombinant mouse $\alpha$-synuclein fibrils or $3 \mu \mathrm{L}$ of saline into striatum. For in vivo evaluation of ligands by two-photon laser scanning, mice anesthetized with $1.5 \%(\mathrm{v} / \mathrm{v})$ isoflurane were unilaterally injected with $3 \mu \mathrm{L}$ of recombinant mouse a-synuclein fibrils into somatosensory cortex (Interaural $1.98 \mathrm{~mm}$, Lateral $2.5 \mathrm{~mm}$, Depth $0.375 \mathrm{~mm}$ ). For double inoculation of $\alpha$-synuclein fibrils and AAV, nine-week-old mice anesthetized with $1.5 \%(\mathrm{v} / \mathrm{v})$ isoflurane were unilaterally injected with $1 \mu \mathrm{L}$ of purified AAV stock into somatosensory cortex (Interaural 1.98 $\mathrm{mm}$, Lateral $2.5 \mathrm{~mm}$, Depth $0.375 \mathrm{~mm}$ ) and $3 \mu \mathrm{L}$ of recombinant mouse $\alpha$ synuclein fibrils into striatum.

In the marmoset, surgeries were performed under aseptic conditions in fully equipped operating suite. We monitored body temperature, heart rate and $\mathrm{SpO}_{2}$ throughout all surgical procedures. The marmoset (2 years old at the time of surgery) was immobilized by intramuscular injection of ketamine ( $25 \mathrm{mg} / \mathrm{kg})$ and xylazine $(2 \mathrm{mg} / \mathrm{kg})$ and then intubated by endotracheal tube. Anesthesia was maintained with isoflurane (1-3\%, to effect). Prior to surgery, MRI $(20 \mathrm{~cm}$ bore, Biospec, Avance-III system; Bruker BioSpin) and X-ray computed tomography (CT) scans (Accuitomo170, J. MORITA CO.) were performed under anesthesia (isoflurane $1-3 \%$, to effect). Overlay MR and CT images were created using PMOD image analysis software (PMOD Technologies Ltd) to estimate stereotaxic coordinates of target brain structures. For injections, the marmoset underwent surgical procedure to open burr holes ( $\sim 3 \mathrm{~mm}$ diameter) for the injection needle. Recombinant marmoset $\alpha$-synuclein fibrils (right hemisphere, total $100 \mu \mathrm{l} ; 50 \mu \mathrm{l} \times$ 
2 regions) and saline (left hemisphere, total $100 \mu \mathrm{l} ; 50 \mu \mathrm{l} \times 2$ regions) were pressure-injected into caudate nucleus (Interaural $9.75 \mathrm{~mm}$ ) and putamen (Interaural $9.75 \mathrm{~mm}$ ) by Hamilton syringe mounted into motorized microinjector (UMP3T-2, WPI) held by manipulator (Model 1460, David Kopf, Ltd.) on a stereotaxic frame.

\section{Ex vivo fluorescence examination}

Mice were anesthetized with $1.5 \%(\mathrm{v} / \mathrm{v})$ isoflurane and intraperitoneally administered BF-227 (1.66 mg/kg) and C05-05 (1.66 mg/kg). 2 hours after administration of BF-227 (25) and 90 min after administration of C05-05, mice were then sacrificed by cervical dislocation, and brains were removed. After quick freezing by powdered dry ice, $20-\mu \mathrm{m}$ thick frozen sections were prepared by cryostat and mounted in non-fluorescent mounting media (VECTASHIELD; Vector Laboratories). Fluorescence images of brain section with no additional staining were captured by DM4000 microscope (Leica) equipped with custom filter cube (excitation band-pass at 414/23 nm and suppression low-pass with 458 $\mathrm{nm}$ cut-off) and BZ-X710 fluorescence microscope (KEYENCE) equipped with Filter set ET-ECFP (Chroma Technology). For immunostaining, sections used for ex vivo examination were fixed in $4 \%$ paraformaldehyde in phosphate buffered saline (PBS) overnight at $4^{\circ} \mathrm{C}$ just prior to staining.

\section{Two-photon laser-scanning microscopy}

For surgical procedure, animals were anesthetized with a mixture of air, oxygen and isoflurane (3-5\% W/V for induction and $2 \%$ W/V for surgery) via a facemask, and a cranial window (4.5-5 $\mathrm{mm}$ in diameter) was attached over the right somatosensory cortex, centered at $2.5 \mathrm{~mm}$ caudal and $2.5 \mathrm{~mm}$ lateral from bregma (52). Two-photon imaging was performed in awake mice two weeks after cranial window surgery at the earliest. Sulforhodamine 101 dissolved in saline (5 $\mathrm{mM}$ ) was intraperitoneally administered ( $4 \mu \mathrm{l} / \mathrm{g}$ body weight) just before initiation of imaging experiments, and $0.05 \mathrm{mg}$ of C05-05, C05-01, and PBB3 dissolved in dimethyl sulfoxide : saline $=1: 1(0.05 \% \mathrm{~W} / \mathrm{V})$ was intraperitoneally administered at various time points. Animals were placed on a custom-made apparatus, and real-time imaging was conducted by two-photon laser scanning microscopy (TCS-SP5 MP, Leica) with an excitation wavelength of 850-900 nm. In evaluation of in vivo labeling of $\alpha$-synuclein inclusions with ligands, two-photon imaging was performed before and 5, 30, 60, and 90 min after administration of ligands. In in 
vivo longitudinal imaging of a-synuclein inclusions with C05-05, two-photon imaging was performed 90 min after administration of C05-05. An emission signal was separated by beam splitter (560/10 nm) and simultaneously detected through band-pass filter for ligands (525/50 nm) and sulforhodamine 101 and mCherry $(610 / 75 \mathrm{~nm})$. A single image plane consisted of $1024 \times 1024$ pixels, with in-plane pixel size of $0.45 \mu \mathrm{m}$. Volume images were acquired up to maximum depth of 200-500 $\mu \mathrm{m}$ from cortical surface with z-step size of $2.5 \mu \mathrm{m}$.

\section{Radiosynthesis}

${ }^{11} \mathrm{C}-\mathrm{PE} 2 \mathrm{I},{ }^{11} \mathrm{C}-\mathrm{PBB} 3,{ }^{18} \mathrm{~F}-\mathrm{PM}-\mathrm{PBB} 3$, and ${ }^{18} \mathrm{~F}-\mathrm{C} 05-01$ were radiosynthesized using their desmethyl, tosylate or nitro precursors as described previously (13, $18,43,53,54)$. Radiolabeling of ${ }^{18} \mathrm{~F}-\mathrm{C} 05-05$ was accomplished by ring-opening reaction of (rac)- ${ }^{18} \mathrm{~F}$-epifluorohydrin with a phenolic precursor (C05-03) in the presence of $1.0 \mathrm{M} \mathrm{NaOH}$ and dimethylformamide at $130^{\circ} \mathrm{C}$ for $20 \mathrm{~min}$ as described in Supplementary Fig. 6 (55). After fluoroalkylation, the crude reaction mixture was transferred into a reservoir for preparative HPLC using Atlantis Prep T3 column (10 × $150 \mathrm{~mm}$, Waters) with a mobile phase consisting of acetonitrile/water (25/75) with $0.1 \%$ trifluoroacetic acid $(\mathrm{v} / \mathrm{v})$ at a flow rate of 5 $\mathrm{mL} / \mathrm{min}$. The fraction corresponding to ${ }^{18} \mathrm{~F}-\mathrm{C} 05-05$ was collected in a flask containing $100 \mu \mathrm{L}$ of $25 \%$ ascorbic acid solution and Tween 80 , and was evaporated to dryness under a vacuum. The residue was dissolved in $3 \mathrm{~mL}$ of saline ( $\mathrm{pH} 7.4$ ) to obtain ${ }^{18} \mathrm{~F}-\mathrm{C} 05-05$. The final formulated product was chemically and radiochemically pure $(>95 \%)$ as detected by analytical HPLC using Atlantis Prep T3 column $(4.6 \times 150 \mathrm{~mm}$, Waters $)$ with mobile phase consisting of acetonitrile/water $(30 / 70)$ with $0.1 \%$ trifluoroacetic acid $(\mathrm{v} / \mathrm{v})$ at a flow rate of 1 $\mathrm{mL} / \mathrm{min}$. Molar activity of ${ }^{18} \mathrm{~F}-\mathrm{C} 05-05$ at the end of synthesis was $218-260$ $\mathrm{GBq} / \mu \mathrm{mol}$, and ${ }^{18} \mathrm{~F}-\mathrm{C} 05-05$ maintained its radioactive purity exceeding $90 \%$ for over 3 hours after formulation.

\section{PET imaging}

PET scans were performed by microPET Focus 220 scanner (Siemens Medical Solutions). Mice were anesthetized with 1.5-2.0\% isoflurane during all PET procedures. Emission scans were acquired for 90 and $120 \mathrm{~min}$ in 3D list mode with an energy window of 350-750 keV immediately after intravenous injection of ${ }^{18} \mathrm{~F}-\mathrm{C} 05-01(23.5 \pm 0.2 \mathrm{MBq})$ and ${ }^{18} \mathrm{~F}-\mathrm{C} 05-05(30.8 \pm 0.4 \mathrm{MBq})$, respectively. Images were reconstructed by either maximum a posteriori methods or filtered 
back projection (FBP) using a Hanning filter cut-off at Nyquist frequency $\left(0.5 \mathrm{~mm}^{-}\right.$ $\left.{ }^{1}\right)$. All image data were subsequently analyzed using PMOD software (PMOD Technologies). For spatial alignment of PET images, template MRI images generated previously (56) were used in this study. Volumes of interest (VOIs) were manually placed on the striatum, cortex, amygdala and cerebellum. The marmoset was anesthetized with $1-3 \%$ isoflurane during all PET procedures. Transmission scans were performed for about 20 min with a Ge-68 source. Emission scans were acquired for $120 \mathrm{~min}$ and $90 \mathrm{~min}$ in 3D list mode with an energy window of $350-750 \mathrm{keV}$ after intravenous bolus injection of ${ }^{18} \mathrm{~F}-\mathrm{C} 05-05$ $(89.6 \pm 15.3 \mathrm{MBq})$ and ${ }^{11} \mathrm{C}-\mathrm{PE} 2 \mathrm{I}(89.2 \pm 2.0 \mathrm{MBq})$, respectively. PET images were reconstructed with FBP. All image data were subsequently analyzed using PMOD software. VOIs were placed on the caudate nucleus, putamen and cerebellum with reference to standard marmoset brain MR image (57).

After anatomical standardization, decay-corrected time-activity curves in each of the VOls were generated as the regional concentration of radioactivity averaged across the specific time window after radioligand injection. In the striatum and cortex, uptake value ratio to the cerebellum was calculated. To quantify ${ }^{11} \mathrm{C}$-PE2I binding, BP ${ }_{N D}$ was calculated with a simplified reference tissue model using the cerebellum as a reference region, and the caudate nucleus and the putamen as signal-rich regions.

\section{Histological examination}

Mice were deeply anesthetized and sacrificed by saline perfusion, and brains were subsequently dissected and fixed in $4 \%$ paraformaldehyde in PBS overnight at $4^{\circ} \mathrm{C}$. After cryo-protection in PBS containing $20 \%$ sucrose, brains were embedded and frozen in OCT compound (SaKuRa), and 20- $\mu \mathrm{m}$ thick fixed frozen sections were prepared by cryostat. The marmoset was deeply anesthetized with an overdose of sodium pentobarbital ( $80 \mathrm{mg} / \mathrm{kg}$, intravenous administration) and transcardially perfused with saline at $4^{\circ} \mathrm{C}$, followed by $4 \%$ paraformaldehyde in $\mathrm{PBS}, \mathrm{pH} 7.4$. The brain was removed from the skull, postfixed in the same fresh fixative overnight, saturated with $30 \%$ sucrose in phosphate buffer at $4{ }^{\circ} \mathrm{C}$, and then cut serially into $40-\mu \mathrm{m}$-thick sections on a freezing microtome. For fluorescence labeling with ligands, mouse fixed frozen sections, marmoset fixed sections, 6- $\mu \mathrm{m}$ thick deparaffinized postmortem human brain sections and $20-\mu \mathrm{m}$ thick fresh frozen human brain sections post-fixed in $4 \%$ paraformaldehyde in PBS were incubated in $20 \%$ and $50 \%$ ethanol containing $30 \mu \mathrm{M}$ ligand, 
respectively, at room temperature for $30 \mathrm{~min}$. The samples were rinsed with $20 \%$ or $50 \%$ ethanol for $5 \mathrm{~min}$, dipped into distilled water twice for $3 \mathrm{~min}$ each, and mounted in VECTASHIELD. Fluorescence images were captured by a DM4000 microscope equipped with a custom filter cube and a BZ-X710 fluorescence microscope equipped with Filter set ET-ECFP. Sections labeled with ligands in fluorescence microscopy, ex vivo examination and autoradiography and their adjacent sections were immunostained with pS129, LB509, pS199/202, and AT8 antibodies with antigen retrieval by autoclaving, and with 6E10 antibodies with antigen retrieval by formic acid. Immunolabeling was then examined using DM4000 and BZ-X710. Images were analyzed using ImageJ software (NIH Image).

\section{Autoradiography}

In vitro autoradiography was performed using 6- $\mu$ m-thick deparaffinized sections derived from MSA brains, 20- $\mu$ m-thick fresh frozen sections post-fixed in $4 \%$ paraformaldehyde in PBS derived from DLB and PDD brains, and 40- $\mu \mathrm{m}-$ thick fixed sections derived from $\alpha$-Syn marmoset. For labeling with ${ }^{18} \mathrm{~F}-\mathrm{C} 05-05$, sections were pre-incubated in $50 \mathrm{mM}$ Tris- $\mathrm{HCl}$ buffer, $\mathrm{pH} 7.4$, containing $20 \%$ ethanol at room temperature for $30 \mathrm{~min}$, and incubated in $50 \mathrm{mM}$ Tris- $\mathrm{HCl}$ buffer, $\mathrm{pH} 7.4$, containing $20 \%$ ethanol and ${ }^{18} \mathrm{~F}-\mathrm{C} 05-05$ (10 nM; molar activity: 260 $\mathrm{GBq} / \mu \mathrm{mol})$ at room temperature for $60 \mathrm{~min}$. Excess concentration $(10 \mu \mathrm{M})$ of C0505 was added to the reaction to determine non-specific radioligand binding. The samples were then rinsed with ice-cold Tris- $\mathrm{HCl}$ buffer containing $20 \%$ ethanol twice for $2 \mathrm{~min}$, and dipped into ice-cold water for $10 \mathrm{sec}$. The sections labeled with ${ }^{18} \mathrm{~F}-\mathrm{C} 05-05$ were subsequently dried with warm air, and exposed to an imaging plate (BAS-MS2025; Fuji Film). Ex vivo autoradiography was performed in mice used in PET imaging. Mice were anesthetized with $1.5 \%(\mathrm{v} / \mathrm{v})$ isoflurane and intravenously administered ${ }^{18} \mathrm{~F}-\mathrm{C} 05-05(27.8 \pm 0.2 \mathrm{MBq}$, molar activity: 63 $\mathrm{GBq} / \mu \mathrm{mol}) .90 \mathrm{~min}$ after administration of ${ }^{18} \mathrm{~F}-\mathrm{C} 05-05$, mice were then sacrificed by cervical dislocation, and the brains were removed. After quick freezing with powdered dry ice, 20- $\mu \mathrm{m}$ thick frozen sections were prepared by cryostat and exposed to an imaging plate. The imaging plate was scanned by BAS-5000 system (Fuji Film) to acquire autoradiograms. Images were analyzed using Multi Gauge software (Fuji Film).

\section{In vitro binding assay}


Frozen tissues derived from the amygdala of a DLB patient and the frontal cortex of an $\mathrm{AD}$ patient were homogenized in $50 \mathrm{mM}$ Tris- $\mathrm{HCl}$ buffer, $\mathrm{pH} 7.4$, containing protease inhibitor cocktail (cOmplete ${ }^{T M}$, EDTA-free; Roche), and stored at $-80^{\circ} \mathrm{C}$ until analyses. To assay radioligand binding with homologous blockade, these homogenates (100 $\mu \mathrm{g}$ tissue) were incubated with $5 \mathrm{nM}{ }^{18} \mathrm{~F}-\mathrm{C} 05-$ 05 (molar activity: $63 \mathrm{GBq} / \mu \mathrm{mol}$ ), $5 \mathrm{nM}{ }^{11} \mathrm{C}-\mathrm{PBB} 3$ (molar activity: $93.4 \pm 24$ $\mathrm{GBq} / \mu \mathrm{mol}$ ), or $1 \mathrm{nM}{ }^{18} \mathrm{~F}-\mathrm{PM}-\mathrm{PBB} 3$ (molar activity: $183.3 \pm 89.2 \mathrm{GBq} / \mu \mathrm{mol}$ ) in the absence or presence of non-radiolabeled C05-05, PBB3, or PM-PBB3 at varying concentrations ranging from $1 \times 10^{-11}$ to $5 \times 10^{-7} \mathrm{M}$ in Tris- $\mathrm{HCl}$ buffer containing $10 \%$ ethanol, $\mathrm{pH} 7.4$, for $30 \mathrm{~min}$ at room temperature. Non-specific binding of ${ }^{18} \mathrm{~F}-\mathrm{C} 05-$ $05,{ }^{11} \mathrm{C}-\mathrm{PBB} 3$, and ${ }^{18} \mathrm{~F}-\mathrm{PM}-\mathrm{PBB} 3$ was determined in the presence of $5 \times 10^{-7} \mathrm{M}$ C05-05, PBB3, and PM-PBB3, respectively. Samples were run in quadruplicate. To assay radioligand binding with heterologous blockade, the DLB amygdala and $\mathrm{AD}$ frontal cortex homogenates (100 $\mu \mathrm{g}$ tissue) were incubated with $5 \mathrm{nM}{ }^{18} \mathrm{~F}$ C05-05 (molar activity: $201 \mathrm{GBq} / \mu \mathrm{mol}$ ) in the absence or presence of clorgiline and selegiline at varying concentrations ranging from $1 \times 10^{-11}$ to $5 \times 10^{-7} \mathrm{M}$ in Tris$\mathrm{HCl}$ buffer containing $10 \%$ ethanol, $\mathrm{pH} 7.4$, for $30 \mathrm{~min}$ at room temperature. Concentration of the competitor inducing 50\% inhibition (IC50) was determined by using non-linear regression to fit a concentration-binding plot to one-site and two-site binding models derived from the Cheng-Prusoff equation with GraphPad Prism version 5.0 (GraphPad Software), followed by F-test for model selection.

\section{Radiometabolite analysis in mice}

${ }^{18} \mathrm{~F}-\mathrm{C} 05-05$ (23.2 $\pm 6.2 \mathrm{MBq}$, molar activity: $\left.320.5 \pm 10.6 \mathrm{GBq} / \mu \mathrm{mol}\right)$ was intravenously administered to awake C57BL/6J mice (male, six-month-old). Mice were decapitated at 5, 30, 60, and $90 \mathrm{~min}$ after ${ }^{18} \mathrm{~F}-\mathrm{C} 05-05$ administration, and blood and brain samples were collected. For blood samples, the whole blood was centrifuged at $15,000 \times \mathrm{g}$ for $2 \mathrm{~min}$ at $4^{\circ} \mathrm{C}$. The resulting plasma $(100 \mu \mathrm{L})$ was mixed with a $150 \mu \mathrm{L}$ of acetonitrile/Methanol (100/50) solution and centrifuged at $15,000 \times \mathrm{g}$ for $2 \mathrm{~min}$ at $4^{\circ} \mathrm{C}$ for deproteinization. An aliquot of the supernatant was then collected for HPLC analysis. For brain samples, the whole brain was homogenized in a $1 \mathrm{~mL}$ of ice-cold saline. The resulting homogenate (100 $\mu \mathrm{L})$ was mixed with a $150 \mu \mathrm{L}$ of acetonitrile/Methanol (100/50) solution and centrifuged at $15,000 \times \mathrm{g}$ for $2 \mathrm{~min}$ at $4^{\circ} \mathrm{C}$ for deproteinization. The supernatant of each brain homogenate was subjected to HPLC analysis. The radiometabolites in the plasma and brain samples were analyzed by an HPLC system (JASCO 
Corporation) equipped with a high-sensitive positron detector (Ohyo Koken Kogyo). The column used was CAPCELL PAK C18 UG80 (5 $\mu \mathrm{m}, 4.6 \mathrm{~mm}$ i.d. x $250 \mathrm{~mm}$, OSAKA Soda). The mobile phase was acetonitrile/50 mM ammonium acetate solution (55/45) at an isocratic condition, and the flow rate was 1.0 $\mathrm{mL} / \mathrm{min}$. The retention time of radioactivity corresponding to that of standard C0505 detected at $390 \mathrm{~nm}$ was identified to be of ${ }^{18} \mathrm{~F}-\mathrm{C} 05-05$. The fractions (\%) of peak area corresponding to ${ }^{18} \mathrm{~F}-\mathrm{C} 05-05$ and radiometabolites were calculated with decay correction.

\section{Molecular docking}

Structure of type I (PDB ID: 6XYO) and Type II (PDB IDs: 6XYP and 6XYQ) filaments of $\alpha$-synuclein from MSA was taken from Protein Data Bank (6). Hydrogens were added and energy minimized by protein preparation wizard of Maestro. Ligand structures were modeled using program LigPrep. Ligands were first docked blindly to five type I a-synuclein filaments by AutoDock Vina (58). A total of 200 docking conformations were generated and analyzed. From this blind docking, 14 binding regions were identified and then ligands were docked into each identified binding site individually. Docking in each binding site was performed by standard precision docking protocol of program Glide. Highest scoring binding pose of each ligand was analyzed and have been reported. Since site S10 and S11 are very close to each other, docking in these two was performed together in single docking run and labeled as S10-11. Binding site S8 and S12 were identified as most likely binding sites and were further analyzed in both the structures of type II filament. Docking scores were converted to affinities $\left(K_{d}\right)$ using relation Score $=-R T \ln \left(K_{d}\right)$. S12 and $S 8$ were compared by superimposing structures in PyMol.

\section{Statistical Analysis}

Statistical significance of the data was analyzed with GraphPad Prism version 5.0. For comparison of multiple groups, data were analyzed by one-way ANOVA with post-hoc Tukey's HSD test. For comparison of multiple groups and regions, data were analyzed by two-way repeated-measures ANOVA with Bonferroni's post hoc analysis.

\section{Data availability}

The data that support the findings of this study are available from the 
corresponding authors upon reasonable request.

\section{Code availability}

The code to generate the results and the figures of this study are available from the corresponding authors upon reasonable request.

\section{Acknowledgments}

The authors thank Kana Osawa, Kaori Yuki, Kanami Ebata, Takahiro Shimizu, Azusa Ishikawa, Tomomi Kokufuta, Jun Kamei, Ryuji Yamaguchi, Yuichi Matsuda, Yoshio Sugii, Anzu Maruyama, and Takashi Okauchi at the National Institutes for Quantum and Radiological Science and Technology for technical assistance. We thank Shunsuke Koga and Dennis W. Dickson at the Mayo Clinic, and John Robinson, John Q. Trojanowski, and Virginia M.-Y. Lee at the University of Pennsylvania for case selection and kindly sharing postmortem brain tissues. This study was supported in part by MEXT KAKENHI Grant Number JP16K19872 to M.O., and by AMED under Grant Number JP18dm0207018 and JP21dm0207072 to M.Higuchi, JP21dk027046 to M.O..

\section{Author contributions}

M.O. and M.Higuchi conceived and designed the project. M.O., M.T., M.F., W.M., Y.N., K.M., K.K., T.K., T.Minamihisamatsu, S.U., S.K.M., Y.Y., H.E., and Y.T. performed the experimental work. A.S., M.S., H.T., H.S., A.K., and M.Hasegawa provided the resource. T.Minamimoto, N.S., M.-R.Z., M.Hasegawa, and M.Higuchi directed the work. All authors reviewed and edited the manuscript.

\section{Competing interests}

M.O., M.-R.Z., and M.Higuchi filed a patent on compounds related to the present report (2019-034997, PCT/JP2020/002607).

\section{References}

1. Alpha-Synuclein in filamentous inclusions of Lewy bodies from Parkinson's disease and dementia with Lewy bodies. Spillantini, M.G. Crowther, R.A. Jakes, 
R. Hasegawa, M. Goedert, M. Proc. Natl. Acad. Sci. U. S. A. 95, 6469-6473 (1998).

2. Aggregation of alpha-synuclein in Lewy bodies of sporadic Parkinson's disease and dementia with Lewy bodies. Baba, M. Nakajo, S. Tu, P.H. Tomita, T. Nakaya, K. et al. Am. J. Pathol. 152, 879-884 (1998).

3. Alpha-synuclein and neurodegenerative diseases. Goedert, M. Nat. Rev. Neurosci. 2, 492-501 (2001).

4. Accumulation of alpha-synuclein/NACP is a cytopathological feature common to Lewy body disease and multiple system atrophy. Wakabayashi, K. Hayashi, S. Kakita, A. Yamada, M. Toyoshima, Y. et al. Acta Neuropathol. 96, 445-452 (1998).

5. Fiber diffraction of synthetic alpha-synuclein filaments shows amyloid-like cross-beta conformation. Serpell, L.C. Berriman, J. Jakes, R. Goedert, M. Crowther, R.A. Proc. Natl. Acad. Sci. U. S. A. 97, 4897-4902 (2000).

6. Structures of a-Synuclein filaments from multiple system atrophy.

Schweighauser, M. Shi, Y. Tarutani, A. Kametani, F. Murzin, A.G. et al. Nature 585, 464-469 (2020).

7. Pathological a-synuclein transmission initiates Parkinson-like neurodegeneration in nontransgenic mice. Luk, K.C. Kehm, V. Carroll, J. Zhang, B. O’Brien, P. et al. Science 338, 949-953 (2012).

8. Prion-like spreading of pathological a-synuclein in brain. Masuda-Suzukake, M. Nonaka, T. Hosokawa, M. Oikawa, T. Arai, T. et al. Brain 136, 1128-1138 (2013).

9. Staging of brain pathology related to sporadic Parkinson's disease. Braak, $\mathrm{H}$. Del Tredici, K. Rüb, U. de Vos, R.A. Jansen Steur, E.N. et al. Neurobiol. Aging 24, 197-211 (2003). 
10. Accumulation of phosphorylated alpha-synuclein in aging human brain. Saito, Y. Kawashima, A. Ruberu, N.N. Fujiwara, H. Koyama, S. et al. J. Neuropathol. Exp. Neurol. 62, 644-654 (2003).

11. $\alpha$-synuclein pathology in Parkinson's disease and related $\alpha-$ synucleinopathies. Henderson, M.X. Trojanowski, J.Q. Lee, V.M. Neurosci. Lett. 709, 134316 (2019).

12. Imaging brain amyloid in Alzheimer's disease with Pittsburgh Compound-B. Klunk, W.E. Engler, H. Nordberg, A. Wang, Y. Blomqvist, G. et al. Ann. Neurol. 55, 306-319 (2004).

13. Imaging of tau pathology in a tauopathy mouse model and in Alzheimer patients compared to normal controls. Maruyama, M. Shimada, H. Suhara, T. Shinotoh, H. Ji, B. et al. Neuron 79, 1094-1108 (2013).

14. Early clinical PET imaging results with the novel PHF-tau radioligand [F-18]T808. Chien, D.T. Szardenings, A.K. Bahri, S. Walsh, J.C. Mu, F. et al. J. Alzheimers Dis. 38, 171-184 (2014).

15. Characterization of 3 Novel Tau Radiopharmaceuticals, ${ }^{11} \mathrm{C}-\mathrm{RO}-963,{ }^{11} \mathrm{C}-$ RO-643, and ${ }^{18} \mathrm{~F}-\mathrm{RO}-948$, in Healthy Controls and in Alzheimer Subjects.

Wong, D.F. Comley, R.A. Kuwabara, H. Rosenberg, P.B. Resnick, S.M. et al. J. Nucl. Med. 59, 1869-1876 (2018).

16. In Vivo Characterization and Quantification of Neurofibrillary Tau PET Radioligand ${ }^{18} \mathrm{~F}-\mathrm{MK}-6240$ in Humans from Alzheimer Disease Dementia to Young Controls. Betthauser, T.J. Cody, K.A. Zammit, M.D. Murali, D. Converse, A.K. et al. J. Nucl. Med. 60, 93-99 (2019).

17. Tau PET imaging with ${ }^{18} \mathrm{~F}-\mathrm{PI}-2620$ in patients with Alzheimer's disease and healthy controls: a first-in-human study. Mueller, A. Bullich, S. Barret, O. Madonia, J. Berndt, M. et al. J. Nucl. Med. 61, 911-919 (2020). 
18. High-contrast in vivo imaging of tau pathologies in Alzheimer's and nonAlzheimer's disease tauopathies. Tagai, K. Ono, M. Kubota, M. Kitamura, S. Takahata, K. et al. Neuron 109, 42-58 (2021).

19. $\left[{ }^{11} \mathrm{C}\right]$ MODAG-001-towards a PET tracer targeting $\alpha$-synuclein aggregates. Kuebler, L. Buss, S. Leonov, A. Ryazanov, S. Schmidt, F. et al. Eur. J. Nucl. Med. Mol. Imaging 48, 1759-1772 (2021).

20. ${ }^{11} \mathrm{C}$ Radiolabeling of anle253b: a Putative PET Tracer for Parkinson's Disease That Binds to $\alpha$-Synuclein Fibrils in vitro and Crosses the Blood-Brain Barrier. Maurer, A. Leonov, A. Ryazanov, S. Herfert, K. Kuebler, L. et al. ChemMedChem 15, 411-415 (2020).

21. 2-(2-[2-Dimethylaminothiazol-5-yl]ethenyl)-6- (2-[fluoro]ethoxy)benzoxazole: a novel PET agent for in vivo detection of dense amyloid plaques in Alzheimer's disease patients. Kudo, Y. Okamura, N. Furumoto, S. Tashiro, M. Furukawa, K. et al. J. Nucl. Med. 48, 553-561 (2007).

22. In vivo visualization of alpha-synuclein deposition by carbon-11-labelled 2[2-(2-dimethylaminothiazol-5-yl)ethenyl]-6-[2-(fluoro)ethoxy]benzoxacole positron emission tomography in multiple system atrophy. Kikuchi, A. Takeda, A. Okamura, N. Tashiro, M. Hasegawa, T. et al. Brain 133, 1772-1778 (2010).

23. Amyloid-Beta Radiotracer $\left[{ }^{18} \mathrm{~F}\right] \mathrm{BF}-227$ Does Not Bind to Cyroplasmic Glial Inclusions of Postmortem Multiple System Atrophy Brain Tissue. Verdurand, M. Levigoureux, E. Lancelot, S. Zeinyeh, W. Billard, T. et al. Contrast Mol. Imaging 2018, 9165458 (2018).

24. Fluorescence and autoradiographic evaluation of tau PET ligand PBB3 to $\alpha-$ synuclein pathology. Koga, S. Ono, M. Sahara, N. Higuchi, M. Dickson, D.W. Mov. Disord. 32, 884-892 (2017).

25. PBB3 imaging in Parkinsonian disorders: Evidence for binding to tau and other proteins. Perez-Soriano, A. Arena, J.E. Dinelle, K. Miao, Q. McKenzie, J. et al. Mov. Disord. 32, 1016-1024 (2017). 
26. Distinct binding of PET ligands PBB3 and AV-1451 to tau fibril strains in neurodegenerative tauopathies. Ono, M. Sahara, N. Kumata, K. Ji, B. Ni, R. et al. Brain 140, 764-780 (2017).

27. Cryo-EM structures of tau filaments from Alzheimer's disease. Fitzpatrick, A.W.P. Falcon, B. He, S. Murzin, A.G. Murshudov, G. et al. Nature 547, 185190 (2017).

28. Tau Filaments and the Development of Positron Emission Tomography Tracers. Goedert, M. Yamaguchi, Y. Mishra, S.K. Highchi, M. Sahara, N. Front. Neurol. 9, 70 (2018).

29. Structures of filaments from Pick's disease reveal a novel tau protein fold. Falcon, B. Zhang, W. Murzin, A.G. Murshudov, G. Garringer, H.J. et al. Nature 561, 137-140 (2018).

30. Identification and in vitro characterization of C05-01, a PBB3 derivative with improved affinity for alpha-synuclein. Miranda-Azpiazu, P. Svedberg, M.

Higuchi, M. Ono, M. Jia, Z. et al. Brain Res. 1749, 147131 (2020).

31. Propagation of pathological $\alpha$-synuclein in marmoset brain. Shimozawa, A. Ono, M. Takahara, D. Tarutani, A. Imura, S. et al. Acta Neuropathol. Commun. 5, 12 (2017).

32. ${ }^{11} \mathrm{C}$-PE2I and ${ }^{18} \mathrm{~F}$-Dopa PET for assessing progression rate in Parkinson's: A longitudinal study. Li, W. Lao-Kaim, N.P. Roussakis, A.A. Martin-Bastida, A. Valle-Guzman, N. et al. Mov Disord 33, 117-127 (2018).

33. PET analysis of dopaminergic neurodegeneration in relation to immobility in the MPTP-treated common marmoset, a model for Parkinson's disease. Ando, K. Obayashi, S. Nagai, Y. Oh-Nishi, A. Minamimoto, T. et al. PLoS One 7, e46371 (2012).

34. Diagnostic accuracy of parkinsonism syndromes by general neurologists. Joutsa, J. Gardberg, M. Röyttä, M. Kaasinen, V. Parkinsonism Relat. Disord. 20, 840-844 (2014). 
35. Therapeutic approaches to target alpha-synuclein pathology. Brundin, $P$. Dave, K.D. Kordower, J.H. Exp. Neurol. 298, 225-235 (2017).

36. Novel tau filament fold in corticobasal degeneration. Zhang, W. Tarutani, A. Newell, K.L. Murzin, A.G. Matsubara, T. et al. Nature 580, 283-287 (2020).

37. Inclusion formation and neuronal cell death through neuron-to-neuron transmission of alpha-synuclein. Desplats, P. Lee, H.J. Bae, E.J. Patrick, C. Rockenstein, E. et al. Proc. Natl. Aced. Sci. U.S.A. 106, 13010-13015 (2009).

38. Neuron-released oligomeric a-synuclein is an endogenous agonist of TLR2 for paracrine activation of microglia. Kim, C. Ho, D.-H. Suk, J.-E. You, S.

Michael, S. et al. Nat. Commun. 4, 1562 (2013).

39. Dynamic transport and localization of alpha-synuclein in primary hippocampal neurons. Yang, M.L. Hasadsri, L. Woods, W.S. George, J.M. Mol. Neurodegener. 5, 9 (2010).

40. Large a-synuclein oligomers inhibit neuronal SNARE-mediated vesicle docking. Choi, B.K. Choi, M.G. Kim, J.Y. Yang, Y. Lai, Y. et al. Proc. Natl. Acad. Sci. U. S. A. 110, 4087-4092 (2013).

41. a-synuclein multimers cluster synaptic vesicles and attenuate recycling. Wang, L. Das, U. Scott, D.A. Tang, Y. McLean, P.J. et al. Curr. Biol. 24, 23192326 (2014).

42. Biochemical staging of synucleinopathy and amyloid deposition in dementia with Lewy bodies. Deramecourt, V. Bombois, S. Maurage, C.A. Ghestem, A. Drobecq, H. et al. Neuropathol. Exp. Neurol. 65, 278-288 (2006).

43. Radiosynthesis, photoisomerization, biodistribution, and metabolite analysis of ${ }^{11} \mathrm{C}$-PBB3 as a clinically useful PET probe for imaging of tau pathology. Hashimoto, H. Kawamura, K. Igarashi, N. Takei, M. Fujishiro, T. et al. J. Nucl. Med. 55, 1532-1538 (2014). 
44. Multiple system atrophy: a sporadic synucleinopathy. Dickson, D.W. Lin, W. Liu, W.K. Yen, S.H. Brain Pathol. 9, 721-732 (1999).

45. Rosa-Neto, Monoamine oxidase B inhibitor, selegiline, reduces ${ }^{18} \mathrm{~F}$ THK5351 uptake in the human brain. Ng, K. P. Pascoal, T. A. Mathotaarachchi, S. Therriault, J. Kang, M. S. et al. Alzheimers Res. Ther. 9, 25 (2017).

46. Tau positron emission tomography imaging in tauopathies: The added hurdle of off-target binding. Lemoine, L. Leuzy, A. Chiotis, K. Rodriguez-Vieitez, E. Nordberg, A. Alzheimers Dement. (Amst) 10, 232-236 (2018).

47. The tau positron-emission tomography tracer AV-1451 binds with similar affinities to tau fibrils and monoamine oxidases. Vermeiren, C. Motte, P. Viot, D. Mairet-Coello, G. Courade, J. P. et al. Mov. Disord. 33, 273-281 (2018).

48. Comparative In Vitro and In Vivo Quantifications of Pathologic Tau Deposits and Their Association with Neurodegeneration in Tauopathy Mouse Models. Ni, R. Ji, B. Ono, M. Sahara, N. Zhang, M. R. et al. J. Nucl. Med. 59, 960-966 (2018).

49. Neuropathological and genetic correlates of survival and dementia onset in synucleinopathies: a retrospective analysis. Irwin, D.J. Grossman, M.

Weintraub, D. Hurtig, H.I. Duda, J.E. et al. Lancet Neurol. 16, 55-65 (2017). 


\section{References}

50. The effect of fragmented pathogenic alpha-synuclein seeds on prion-like propagation. Tarutani, A. Suzuki, G. Shimozawa, A. Nonaka, T. Akiyama, H. et al. J. Biol. Chem. 291, 18675-18688 (2016).

51. Deschloroclozapine: a potent and selective chemogenetic actuator enables rapid neuronal and behavioral modulations in mice and monkeys. Nagai, Y. Miyakawa, N. Takuwa, H. Hori, Y. Oyama, K. et al. Nat. Neurosci. 23, 11571167 (2020).

52. Long-term, high-resolution imaging in the mouse neocortex through a chronic cranial window. Holtmaat, A. Bonhoeffer, T. Chow, D.K. Chuckowree, J. De Paola, V. et al. Nat. Protoc. 4, 1128-1144 (2009).

53. Correlation between decreased motor activity and dopaminergic degeneration in the ventrolateral putamen in monkeys receiving repeated MPTP administrations: a positron emission tomograohy study. Nagai, Y. Minamimoto, T. Ando, K. Obayashi, S. Ito, H. et al. Neurosci. Res. 73, 61-67 (2012).

54. Radiosynthesis and preliminary evaluation of 4-[ $\left.{ }^{18} \mathrm{~F}\right]$ fluoro- $\mathrm{N}-[4-[6-$ (isopropylamino)pyrimidin-4-yl]-1,3-thiazol-2-yl]-N-methylbenzamide as a new positron emission tomography ligand for metabotropic glutamate receptor subtype 1. Yamasaki, T. Fujinaga, M. Yoshida, Y. Kumata, K. Yui, J. et al. Bioorg. Med. Chem. Lett. 21, 2998-3001 (2011).

55. Automated Synthesis of (rac)-, (R)-, and (S)-[18 F]Epifluorohydrin and Their Application for Developing PET Radiotracers Containing a 3-[ $\left.{ }^{18} \mathrm{~F}\right]$ Fluoro-2hydroxypropyl Moiety. Fujinaga, M. Ohkubo, T. Yamasaki, T. Zhang, Y. Mori, W. et al. ChemMedChem 13, 1723-1731 (2018).

56. Longitudinal, quantitative assessment of amyloid, neuroinflammation, and anti-amyloid treatment in a living mouse model of Alzheimer's disease enabled by positron emission tomography. Maeda, J. Ji, B. Irie, T. Tomiyama, T. Maruyama, M. et al. J. Neurosci. 27, 10957-10968 (2007). 
57. Population-averaged standard template brain atlas for the common marmoset (Callithrix jacchus). Hikishima, K. Quallo, M.M. Komaki, Y. Yamada, M. Kawai, K. et al. Neuroimage 54, 2741-2749 (2011).

58. AutoDock Vina: improving the speed and accuracy of docking with a new scoring function, efficient optimization, and multithreading. Trott, O. and Olson, A.J. J Comput Chem 31, 455-461 (2010). 
a

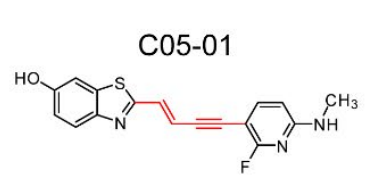

C05-03
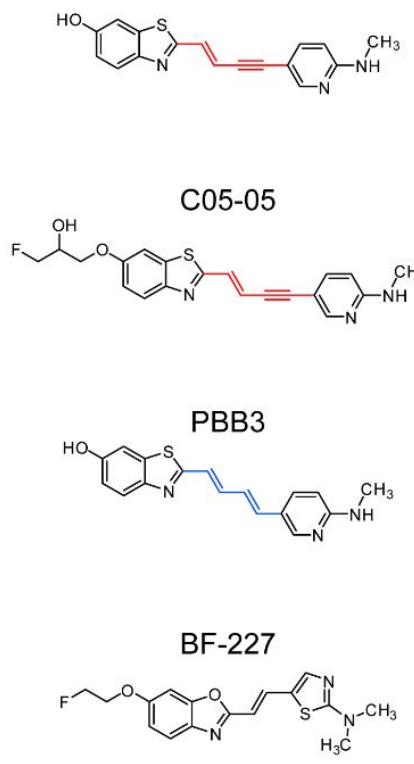

b
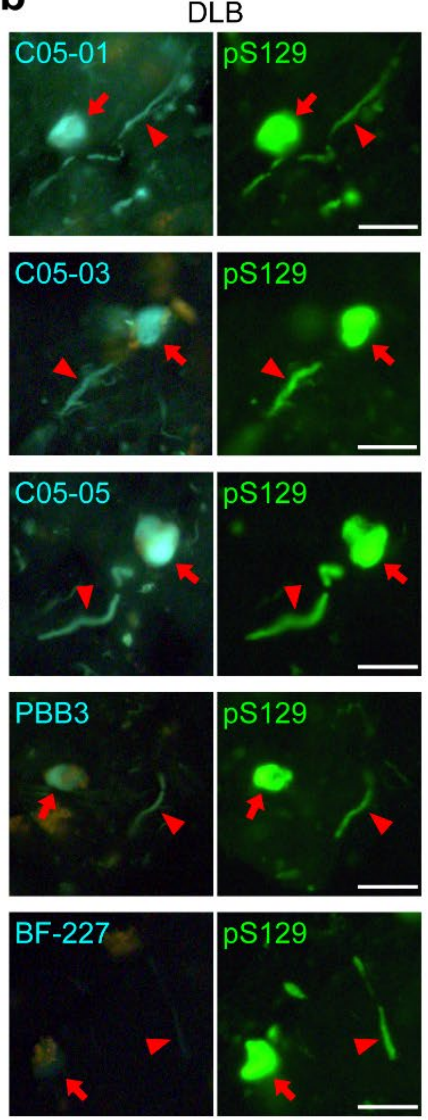

C DLB
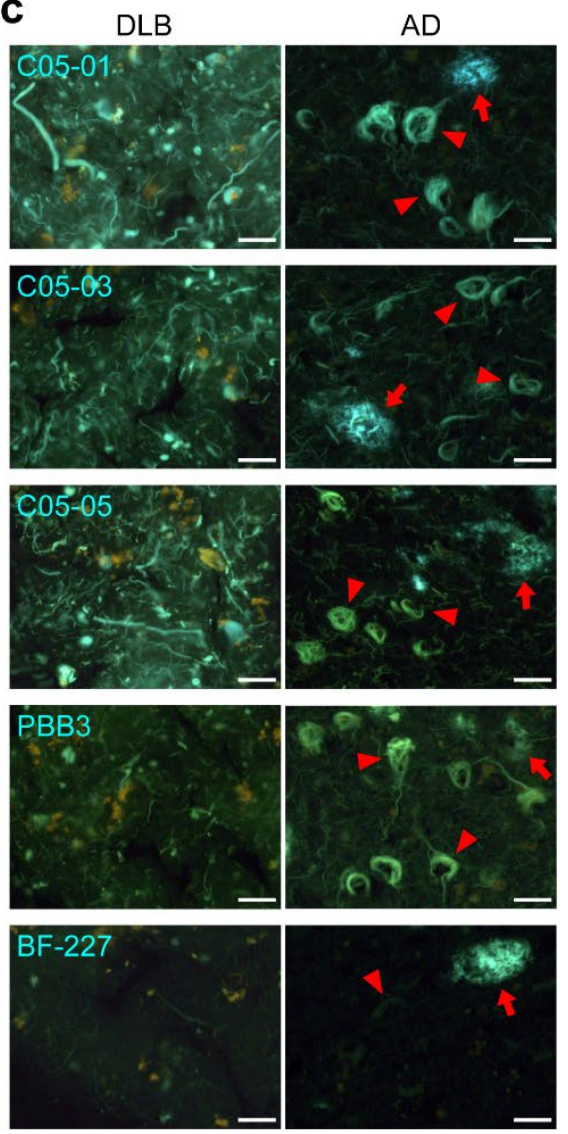

d

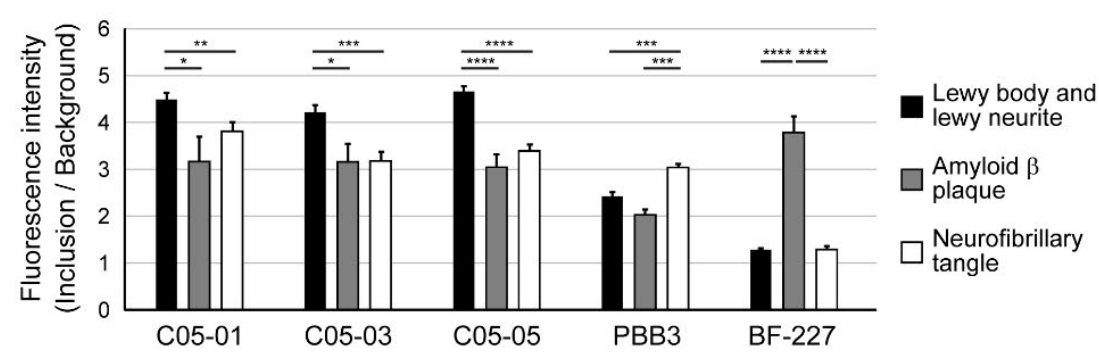

e
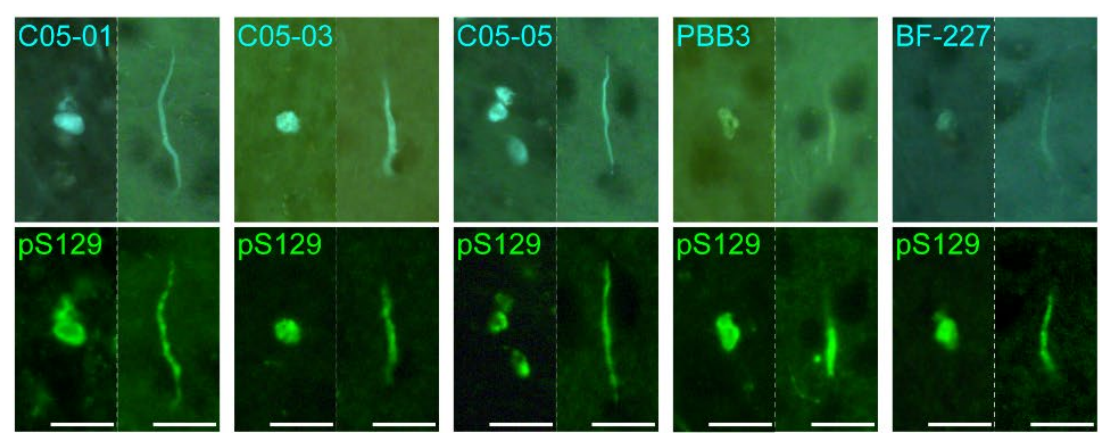

Fig. 1 | C05 series compounds bind to $\alpha$-synuclein inclusions in DLB and mouse model of a-synucleinopathy in vitro.

(a) Chemical structures of C05 series compounds, PBB3 and BF-227. C05-01, 
C05-03, and C05-05 are derivatives from PBB3 with the substitution of its $(2 E, 4 E)$-hexa-2,4-diene linker (blue) with $(E)$-hex-2-en-4-yne (red). (b) Double fluorescence staining of Lewy bodies (arrows) and Lewy neurites (arrowheads) in the amygdala sections of a patient with DLB (also see Supplementary Table 1) with $30 \mu \mathrm{M}$ of self-fluorescent ligands (left) and anti-phosphorylated $\alpha$-synuclein antibody, pS129 (right). C05-01, C05-03, and C05-05 intensely labeled $\alpha-$ synuclein inclusions in DLB brain sections, while PBB3 and BF-227 yielded moderate and weak staining of these lesions, respectively. (c) Fluorescence microscopic images of various fibrillary protein pathologies, including Lewy bodies and Lewy neurites in the amygdala sections of a patient with DLB (left) and amyloid plaques (right, arrows) and neurofibrillary tangles (right, arrowheads) in the middle frontal gyrus sections of a patient with $A D(A D-1$, also see Supplementary Table 1), labeled with C05-01, C05-03, C05-05, PBB3, and BF227 were taken under a uniform imaging condition. (d) Fluorescence signal intensities in Lewy bodies and neurites (black), amyloid plaques (gray), and neurofibrillary tangles (white) in the images illustrated in c were normalized according to background signals. Quantification of the background-corrected fluorescence intensity indicated that $\mathrm{C} 05-01\left(\mathrm{~F}_{(2,74)}=6.729, p=0.0021\right), \mathrm{C} 05-03$ $\left(F_{(2,73)}=9.151, p=0.0003\right)$, and $\operatorname{C05-05}\left(F_{(2,85)}=36.92, p<0.0001\right)$ bound to $\alpha-$ synuclein pathologies produced significantly more intense signals than these chemicals bound to $A \beta$ and tau pathologies. In contrast, PBB3 bound to tau pathologies elicited stronger fluorescence than this compound bound to $\alpha$ synuclein and $A \beta$ pathologies $\left(F_{(2,73)}=12.57, p<0.0001\right)$, and the fluorescence signals attributed to $B F-227$ bound to $A \beta$ pathologies were significantly more intense than the signals related to $\alpha$-synuclein- and tau-bound BF-227 $\left(F_{(2,63)}=\right.$ 114.0, $p<0.0001)$. Data are presented as mean \pm SD. ${ }^{*}, p<0.05 ;{ }^{*}, p<0.01$; ${ }^{* * *}, p<0.001 ;{ }^{* * *}, p<0.0001$ by one-way ANOVA with post-hoc Tukey's HSD test. (e) Double fluorescence staining of $\alpha$-synuclein inclusions resembling Lewy bodies (left) and Lewy neurites (right) in the neocortical sections of an a-Syn mouse injected with $\alpha$-synuclein fibrils into the unilateral striatum (10 weeks after inoculation) with $30 \mu \mathrm{M}$ of self-fluorescent ligands (top) and pS129 (bottom). Scale bars, $20 \mu \mathrm{m}(\mathbf{b}, \mathbf{c}$, and $\mathbf{e})$. 


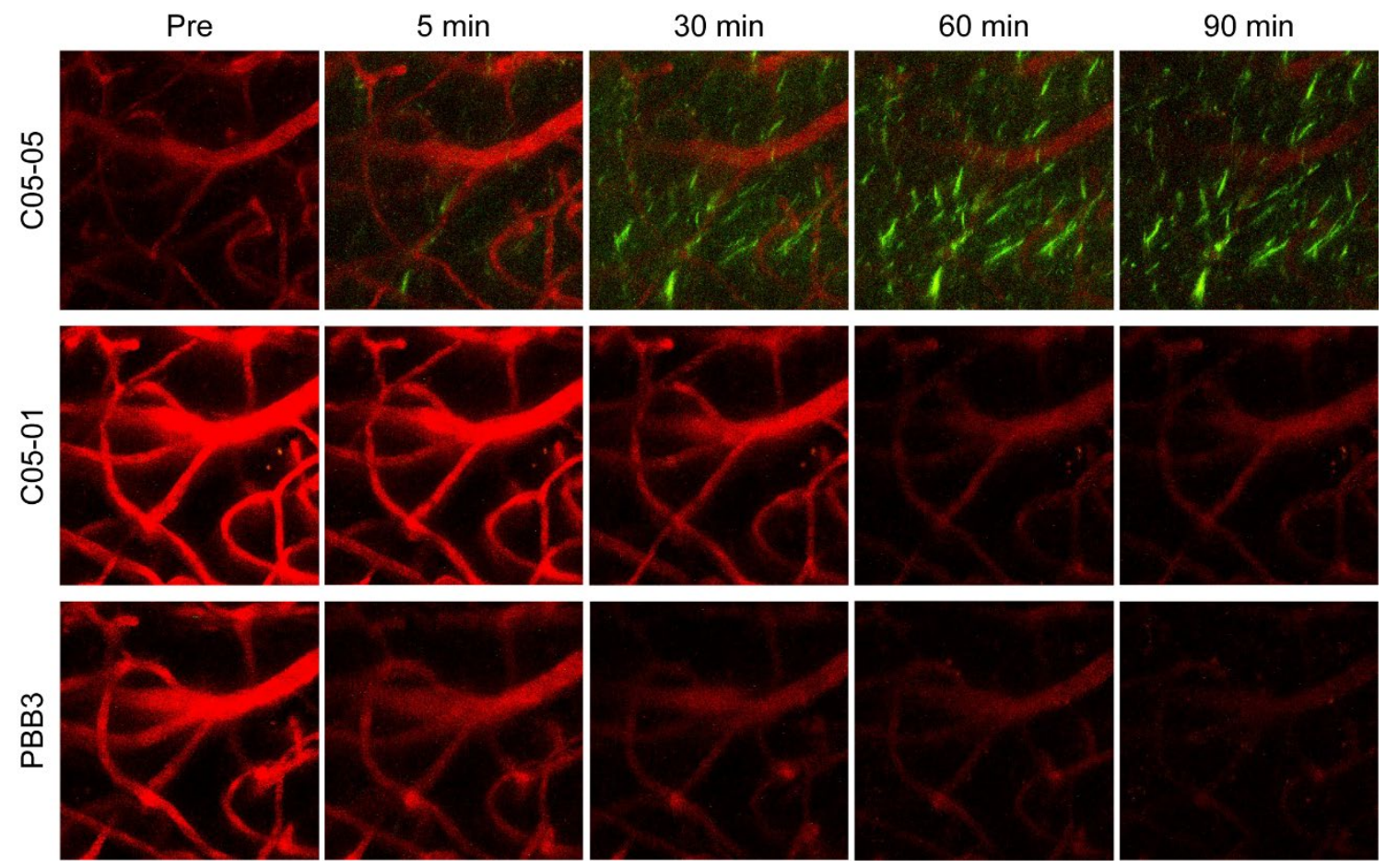

Fig. 2 | C05-05 enables in vivo optical visualization of individual a-synuclein inclusions in the brain of an a-Syn mouse model.

Maximum intensity projection of fluorescence signals in an identical 3D volume (field of view, $150 \times 150 \mu \mathrm{m}$; depth, $25-100 \mu \mathrm{m}$ from the brain surface) of the somatosensory cortex of a living $\alpha-S y n$ mouse at 8 - 10 weeks after inoculation of $\alpha$-synuclein fibrils into the neocortex. Exogenous $\alpha$-synuclein fibrils were found to vanish by 2 weeks after injection, followed by aggregation of endogenous $\alpha$ synuclein molecules. From left, images acquired before (Pre) and 5, 30, 60 and 90 min after intraperitoneal administration of C05-05 (1.66 mg/kg) (top), C05-01 $(1.66 \mathrm{mg} / \mathrm{kg})$ (middle), and PBB3 $(1.66 \mathrm{mg} / \mathrm{kg})$ (bottom) are displayed. Cerebral blood vessels were labeled in red with intraperitoneally administered sulforhodamine 101. Somatodendritic labeling of putative neurons with C05-05 was observed as green fluorescence from 5 min after ligand administration. Fluorescence images of the corresponding area at 5 - 90 min after C05-01 and PBB3 injections demonstrated no overt retention of the tracer in the tissue. 


\section{a}

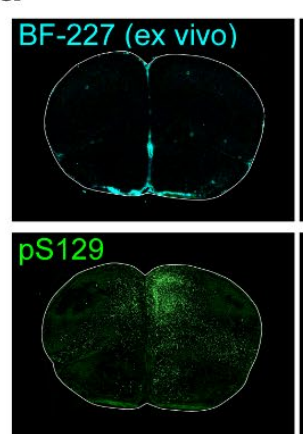

Bregma

$2.22 \mathrm{~mm}$

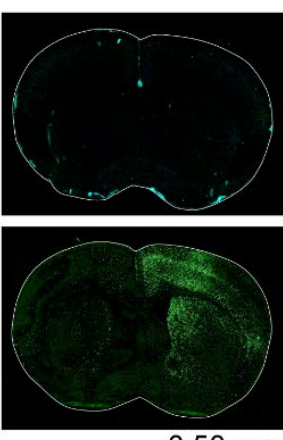

$0.50 \mathrm{~mm}$

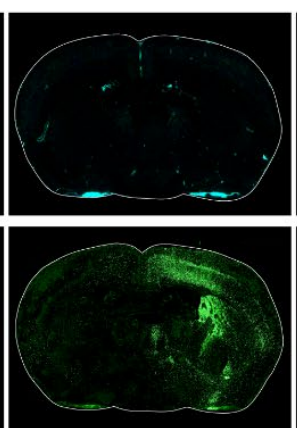

$-0.46 \mathrm{~mm}$

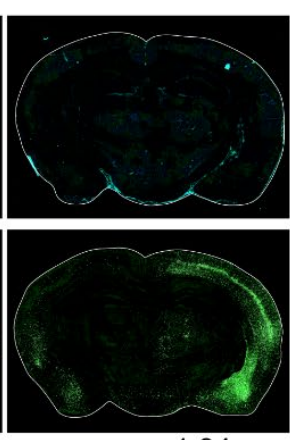

$-1.94 \mathrm{~mm}$
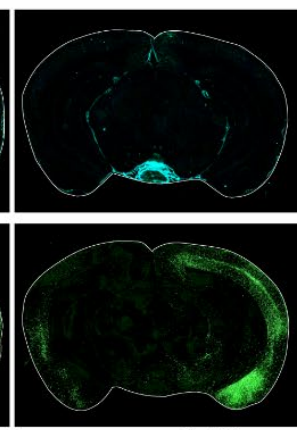

$-3.16 \mathrm{~mm}$

b
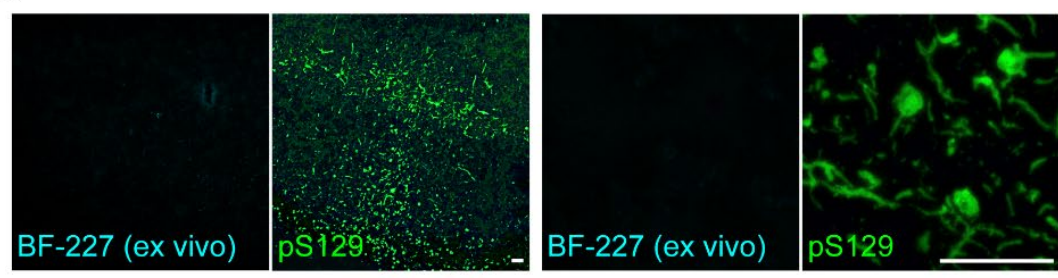

c
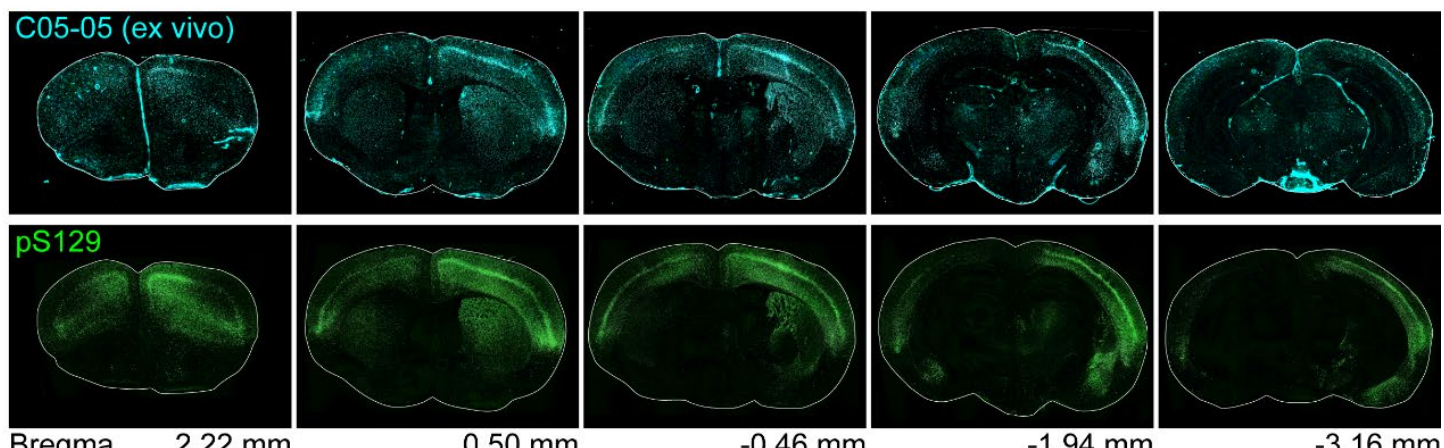

Bregma

$0.50 \mathrm{~mm}$

$-0.46 \mathrm{~mm}$

$-1.94 \mathrm{~mm}$

$-3.16 \mathrm{~mm}$

d
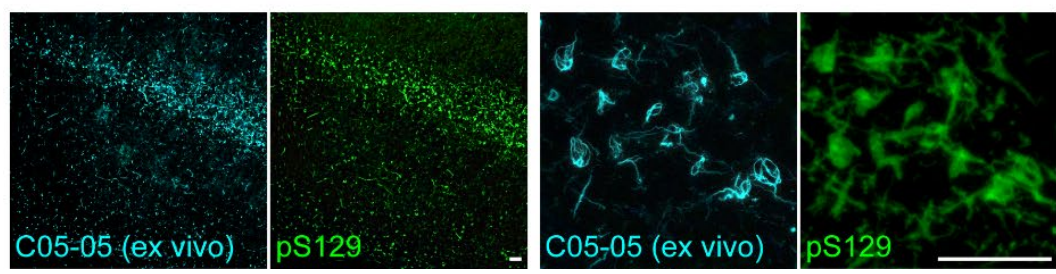

Fig. 3 | C05-05 enables ex vivo detection of $\alpha$-synuclein inclusions in the brain of an $\alpha$-synucleinopathy mouse model.

(a) Ex vivo examination of frozen brain sections from an a-Syn mouse at 10 weeks after the inoculation of a-synuclein fibrils into the right striatum. The brain tissue was collected at 2 hours after intraperitoneal administration of BF-227 
(1.66 mg/kg). Distributions of systemically injected BF-227 in coronal brain sections (top) and postmortem immunolabeling of adjacent sections with pS129 (bottom) at bregma $+2.22,+0.50,-0.46,-1.94$, and $-3.16 \mathrm{~mm}$ are displayed. (b) Medium-power (left) and high-power (right) photomicrographs of cortical sections shown in a. Ex vivo examination revealed that a-synuclein inclusions were devoid of labeling with intraperitoneally administered BF-227. (c) Ex vivo examination of frozen brain sections from an $\alpha$-Syn mouse at 8 weeks after the inoculation of $\alpha$ synuclein fibrils into the right striatum. The brain tissue was collected at $90 \mathrm{~min}$ after intraperitoneal administration of C05-05 (1.66 mg/kg). Distributions of systemically injected $\mathrm{C} 05-05$ in coronal brain sections (top) and immunolabeling of adjacent brain sections with pS129 (bottom) at bregma $+2.22,+0.50,-0.46$, 1.94 and $-3.16 \mathrm{~mm}$ are displayed. (d) Medium-power (left) and high-power (right) photomicrographs of cortical sections shown in c. Individual a-synuclein inclusions were found to be intensely labeled with intraperitoneally administered C05-05. Scale bars, $50 \mu \mathrm{m}$ (b and d). 
a

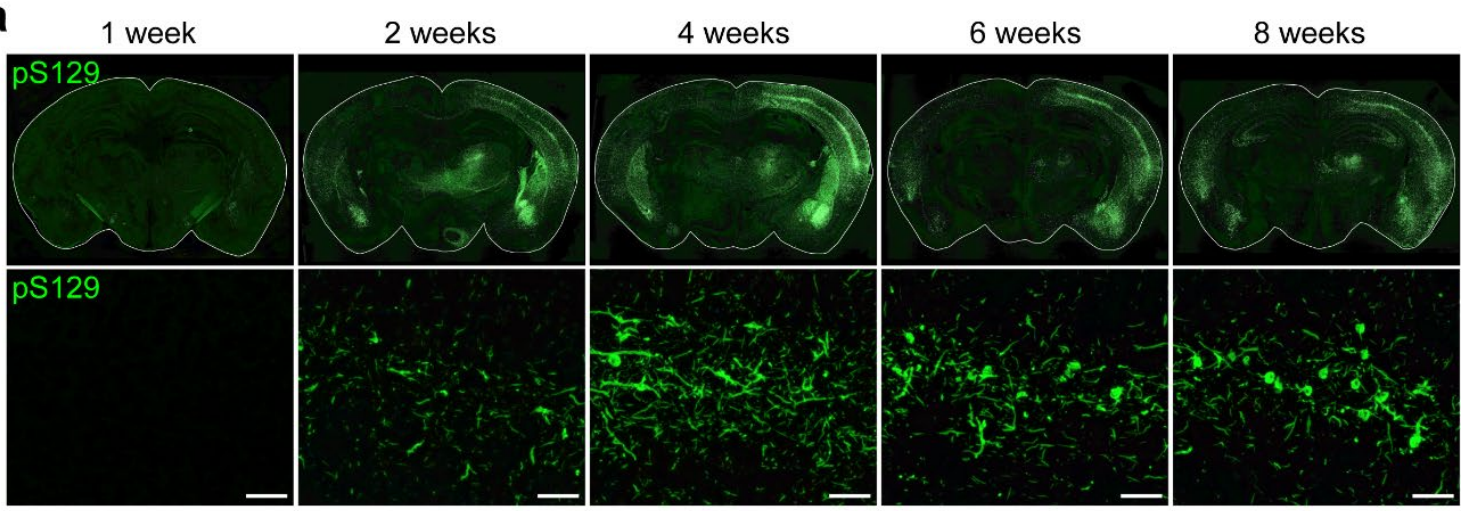

b

2 weeks

4 weeks

6 weeks

8 weeks

12 weeks
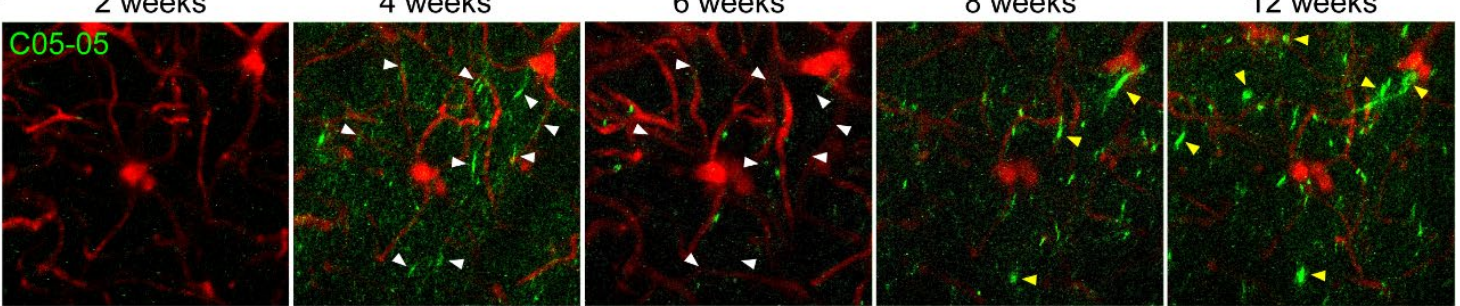

C

+1 week

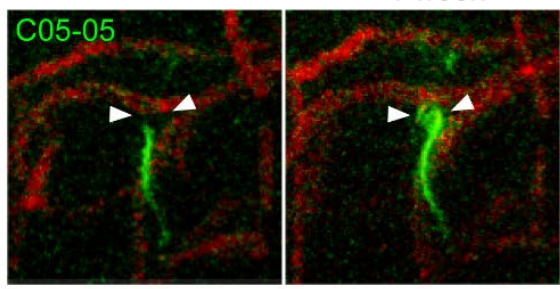

d

+1 week
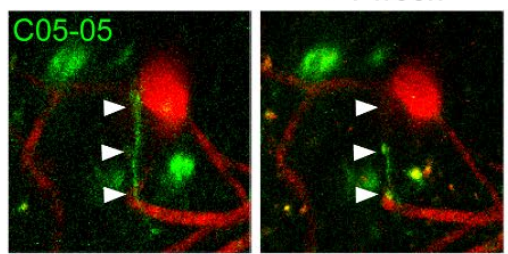

+2 weeks
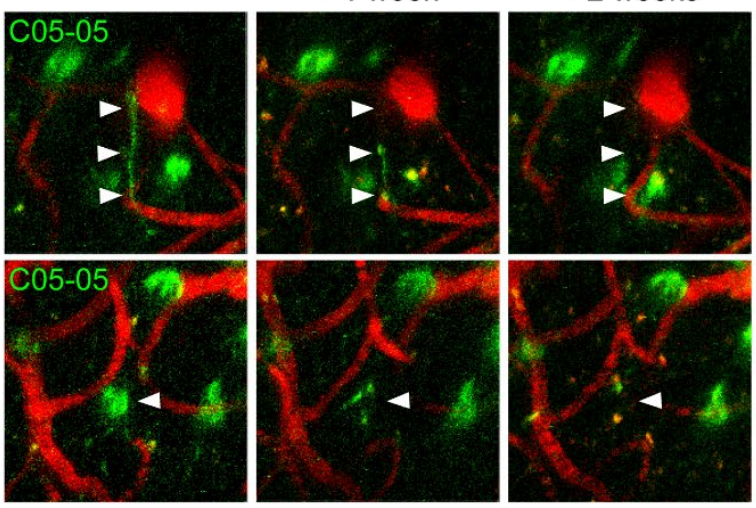

e

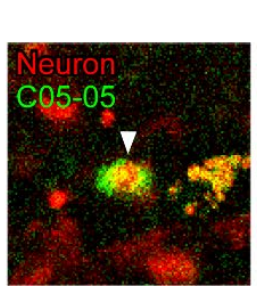

+1 week +3 weeks
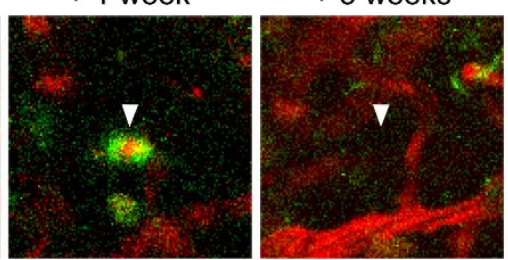

Fig. 4 | Pathological $\alpha$-synuclein propagates to extensive brain areas with a transient oscillation of the aggregate amount in the brains of a living $\alpha$-Syn mouse model.

(a) Distribution of phosphorylated a-synuclein immunostained with pS129 in coronal brain sections at bregma -1.94 of $\alpha$-Syn mice at 1, 2, 4, 6, and 8 weeks 
after inoculation of a-synuclein fibrils into the right striatum (top), and high-power photomicrographs of the ipsilateral somatosensory cortex (bottom). Scale bars, $50 \mu \mathrm{m}$. (b) Longitudinal in vivo two-photon microscopic imaging of a-synuclein inclusions with systemically administered C05-05 in the right somatosensory cortex of a single indivisual $\alpha$-Syn mouse at 2, 4, 6, 8, and 12 weeks after inoculation of $\alpha$-synuclein fibrils into the right striatum. A maximum projection of fluorescence in an identical 3D volume (field of view, $182 \times 182 \mu \mathrm{m}$; depth, 40 $400 \mu \mathrm{m}$ from the brain surface) at $90 \mathrm{~min}$ after intraperitoneal administration of C05-05 demonstrated propagation of C05-05-positive a-synuclein inclusions to the cortical area from 4 weeks after the intrastriatal fibril inoculation, and subsequent changes in the subcellular location and amount of the inclusions. White arrowheads indicate neuritic a-synuclein accumulations which disappeared from 4 to 6 weeks after the fibril inoculation, and yellow arrowheads indicate somatic $\alpha$-synuclein inclusions which appeared from 8 weeks after the fibril inoculation. (c-e) Longitudinal intravital microscopy of the somatosensory cortex (field of view, $55 \times 55 \mu \mathrm{m}$; depth, 0 - $75 \mu \mathrm{m}$ from the brain surface) of an a-Syn mouse demonstrated extension of a C05-05-positive intraneuronal $\alpha$ synuclein inclusion from neurite to soma in a week (c, arrowheads), and disappearance of C05-05-positive (green) neuritic inclusion similar to Lewy neurite (d, top, arrowheads) and somatic deposit resembling Lewy body (d, bottom, arrowheads) like inclusions in two weeks, along with loss of a mCherryexpressing (red) neuron bearing a C05-05-positive (green) inclusion (e, arrowheads) in three weeks. Cerebral blood vessels were also labeled in red with intraperitoneally administered sulforhodamine 101 (b-e). 
a

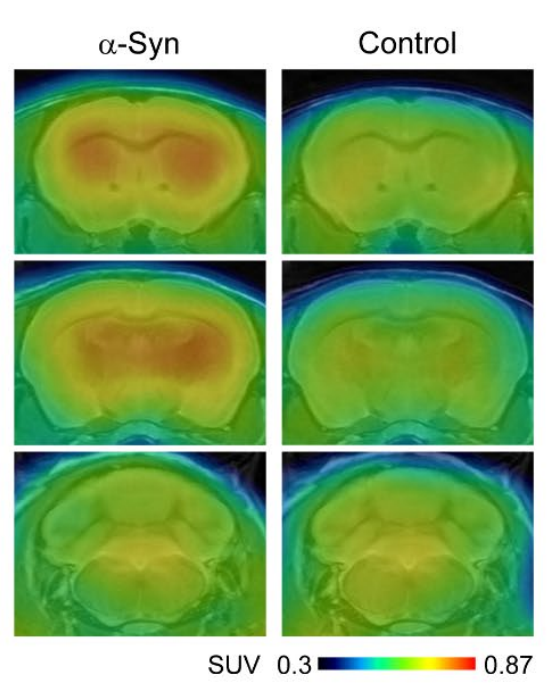

C

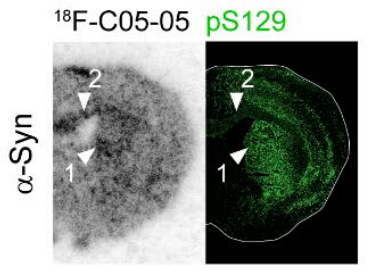

${ }^{18} \mathrm{~F}-\mathrm{C} 05-05$ pS129

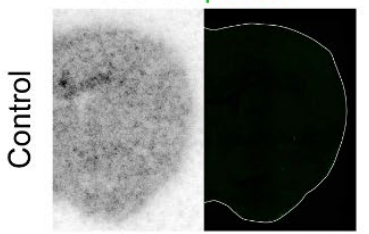

d

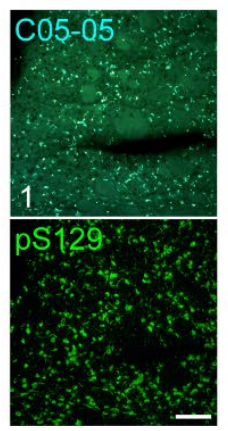

b
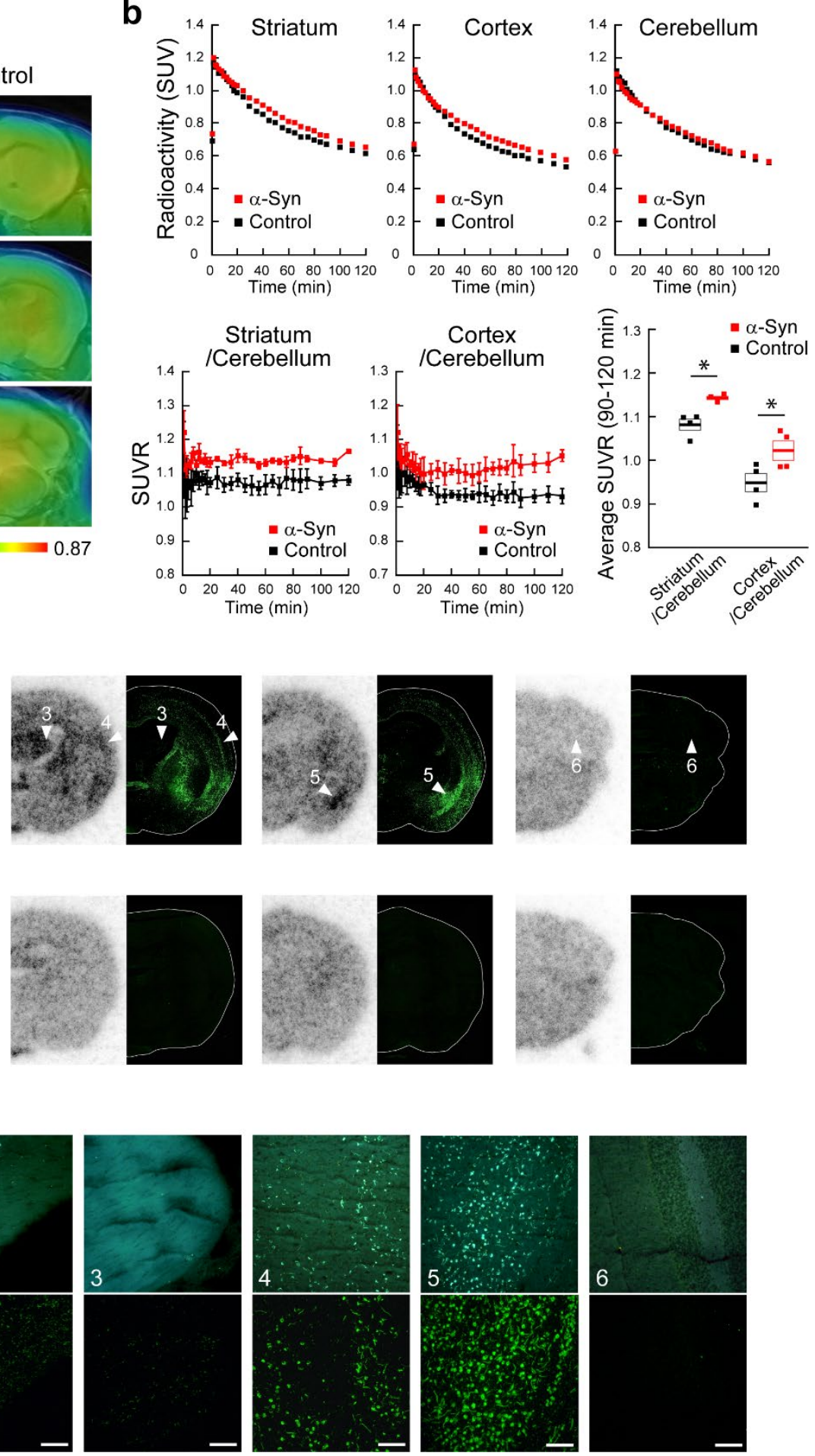

Fig. 5 | In vivo PET imaging with ${ }^{18} \mathrm{~F}-\mathrm{C} 05-05$ detects $\alpha$-synuclein deposits in the brains of $\alpha-S y n$ mice.

(a) Coronal PET images at bregma $+0.50 \mathrm{~mm}$ (top) and $-0.46 \mathrm{~mm}$ (middle) containing the striatum and neocortex, and $-6.64 \mathrm{~mm}$ (bottom) containing the 
cerebellum generated by averaging dynamic scan data at 60 - 90 min after intravenous administration of ${ }^{18} \mathrm{~F}-\mathrm{C} 05-05(30.8 \pm 0.4 \mathrm{MBq})$ in mice at 6 months after inoculation of $\alpha$-synuclein fibrils ( $\alpha$-Syn mouse, left) or saline (control mouse, right) into the bilateral striata. PET images are superimposed on an MRI template. Voxel values represent SUV. (b) Time-radioactivity curves in the striatum, neocortex, and cerebellum during the dynamic PET scan (top), time-course changes in the target-to-cerebellum ratio of radioactivity (SUVR, left and middle panels in bottom row), and the average of target-to-cerebellum ratios at $90-120$ min (bottom, right) in a-Syn (red symbols) and control (black symbols) mice. There were significant main effects of animal group and region in two-way, repeated-measures ANOVA (group, $\mathrm{F}_{(1,6)}=11.39$, $p=0.015$; region, $\mathrm{F}_{(1,6)}=111.9$, $p<0.0001) .{ }^{*}, p<0.05$ by Bonferroni's post hoc test. Data are presented as mean (top) or mean \pm SEM (bottom) in four a-Syn or control mice. (c) Ex vivo examination of frozen brain sections obtained from a-Syn (top) and control (bottom) mice after PET imaging to assess distributions of intravenously administered ${ }^{18} \mathrm{~F}-\mathrm{C} 05-05$ (27.8 $\left.\pm 0.2 \mathrm{MBq}\right)$, in comparison with immunolabeling of the same sections with pS129. From left, coronal brain sections at bregma +0.50, -0.46, -1.94, and $-6.64 \mathrm{~mm}$ are displayed. (d) High-power photomicrographs showing double fluorescence staining of the section used for ex vivo examination with $30 \mu \mathrm{M}$ of C05-05 (top) and pS129 (bottom). Areas correspond to those indicated by arrowheads in C. The striatum (1), somatosensory cortex (4), and amygdala (5) of an a-Syn mouse contained abundant $\alpha$-synuclein inclusions. The corpus callosum (2) and fimbria of the hippocampus (3) showed a small number of $\alpha$-synuclein deposits. The cerebellum (6) contained very few a-synuclein inclusions. Scale bars, $100 \mu \mathrm{m}$. 
a

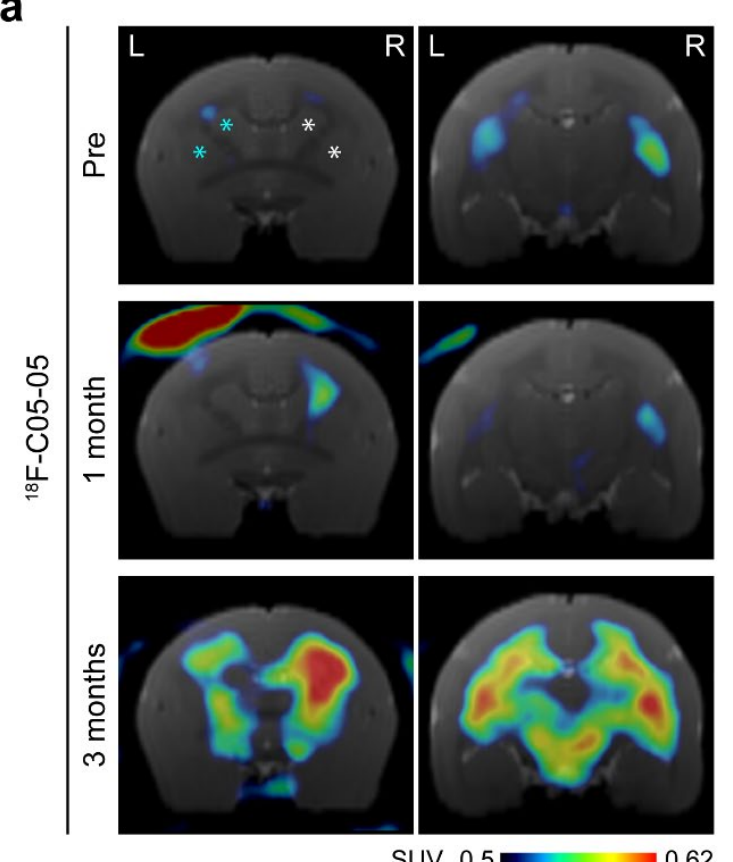

SUV $0.5=0.62$

b

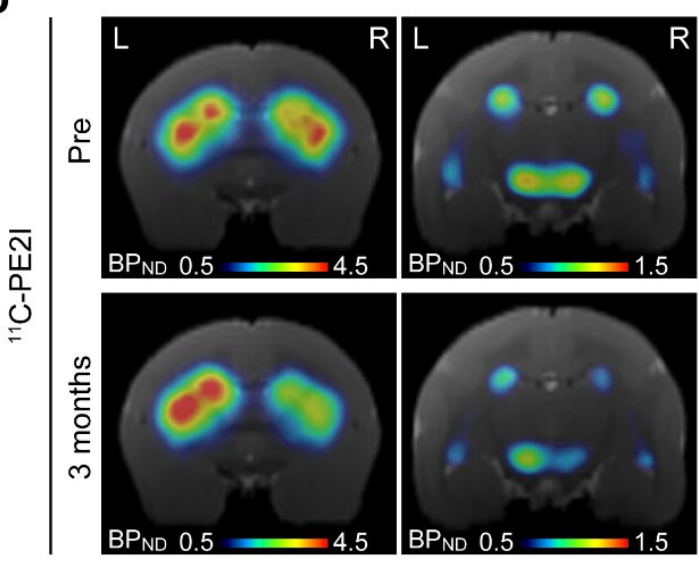

C

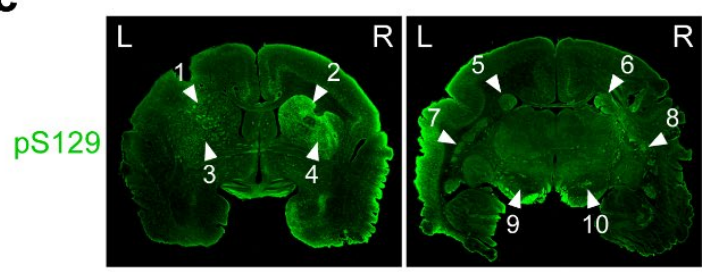

d
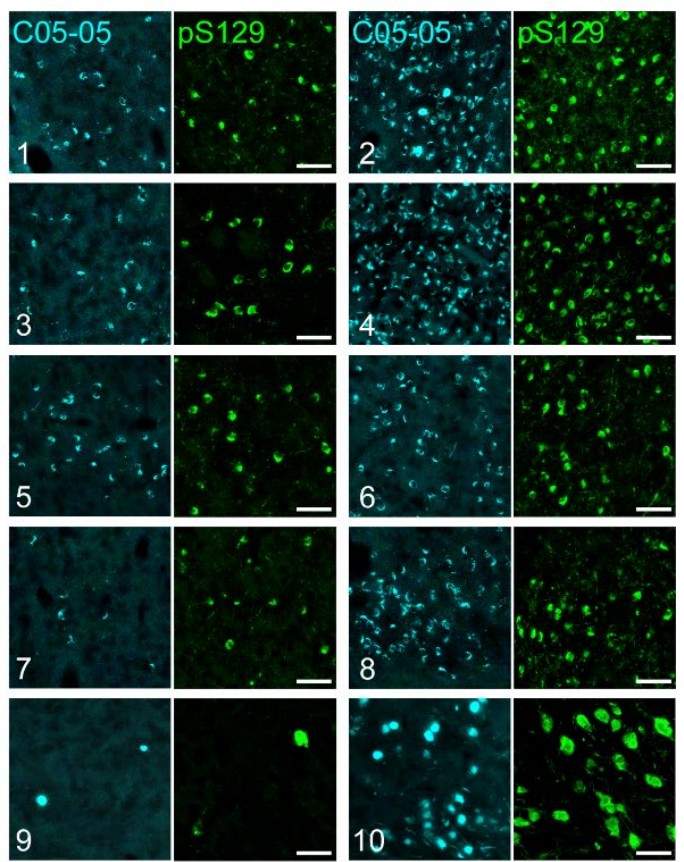

Fig. 6 | Longitudinal in vivo PET imaging with ${ }^{18} \mathrm{~F}-\mathrm{C} 05-05$ visualizes the propagation of pathological $\alpha$-synuclein aggregates in the brain of an $\alpha$-Syn marmoset.

(a) Coronal brain images in a marmoset injected with a-synuclein fibrils and saline into the right and left caudate nucleus and putamen, respectively, generated by averaging dynamic PET data at 30 - 120 min after intravenous administration of ${ }^{18} \mathrm{~F}-\mathrm{C} 05-05$ (89.6 $\pm 15.3 \mathrm{MBq}$ ) (also see Supplementary Fig. 11). Images were acquired before (Pre), and 1 and 3 months after the fibril inoculation, and white and blue asterisks indicate the sites of a-synuclein fibril and saline injections, respectively. Brain volume data were sectioned at $9.5 \mathrm{~mm}$ (left) and $5.0 \mathrm{~mm}$ (right) 
anterior to the interaural line to generate images containing the caudate nucleus/putamen and caudate nucleus/putamen/substantia nigra, respectively. PET images are superimposed on an MRI template, and voxel values represent SUV. Longitudinal ${ }^{18} \mathrm{~F}-\mathrm{C} 05-05-\mathrm{PET}$ showed the expansion of radioactivity signals from a part of the right caudate nucleus to extensive brain areas, including bilateral regions of the caudate nucleus, putamen, and substantia nigra from 1 to 3 months after inoculation. (b) Parametric images of BPND for ${ }^{11} \mathrm{C}-\mathrm{PE} 2 \mathrm{I}$ (radioactivity dose: $89.2 \pm 2.0 \mathrm{MBq}$ ) in a single individual $\alpha$-Syn marmoset demonstrated reduction of the radioligand binding in the right caudate nucleus, putamen, and substantia nigra at 3 months after inoculation compared to the baseline before inoculation (Pre). Brain volume data were sectioned at $9.5 \mathrm{~mm}$ (left) and $5.0 \mathrm{~mm}$ (right) anterior to the interaural line, and BP ${ }_{\mathrm{ND}}$ images were superimposed on an MRI template. (c) Histopathological assays were carried out 1 month after the final PET scan, demonstrating a similarity between the regional distributions of a-synuclein inclusions stained with pS129 and localization of radioligand retentions in ${ }^{18} \mathrm{~F}-\mathrm{C} 05-05-\mathrm{PET}$ images at 3 months. (d) High-power photomicrographs showing fluorescence staining of brain sections shown in $B$ with pS129 and adjacent brain sections with $30 \mu \mathrm{M}$ of C05-05. Areas correspond to those indicated by arrowheads in b. The right caudate nucleus ( 2 and 6 ), putamen (4 and 8), and substantia nigra (10) contained highly abundant $\alpha$ synuclein inclusions. The left caudate nucleus ( 1 and 5 ) and putamen ( 3 and 7 ) contained moderate amounts of a-synuclein deposits, and the left substantia nigra (9) contained sparse a-synuclein inclusions. Scale bars, $50 \mu \mathrm{m}$. 


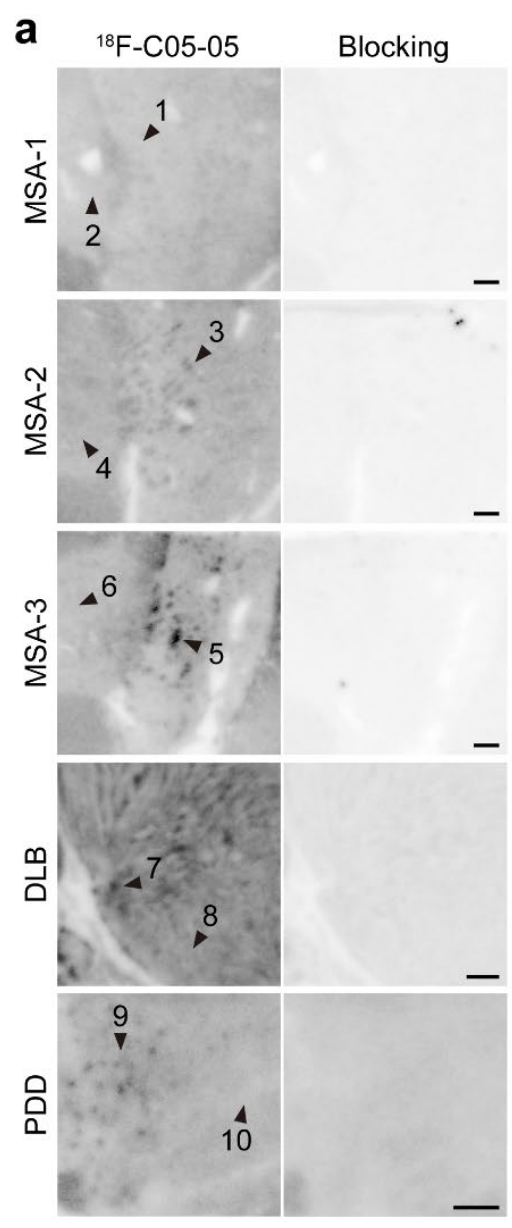

b

C05-05 LB509/pS199/202

C05-05

LB509/pS199/202
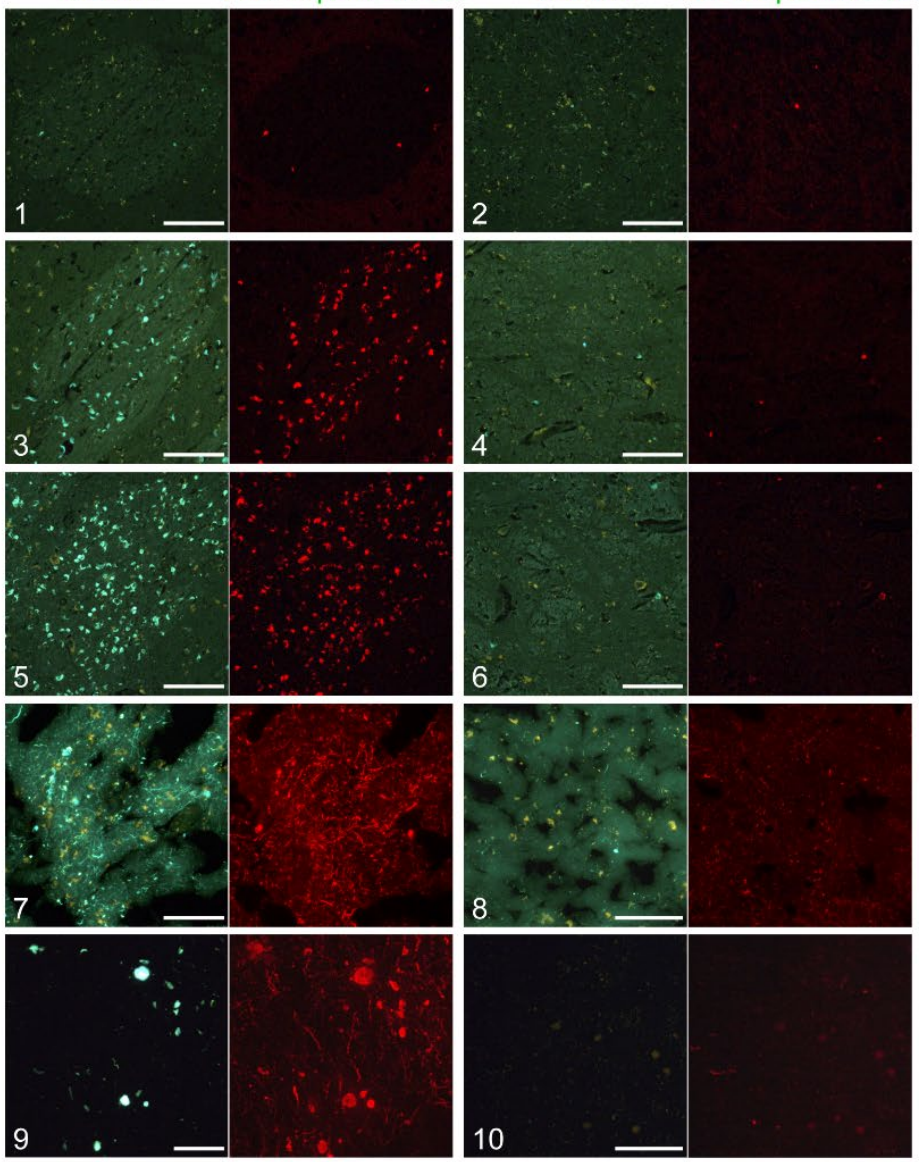

C ${ }^{18} \mathrm{~F}-\mathrm{C} 05-05$

d

e ${ }^{18} \mathrm{~F}-\mathrm{PM}-\mathrm{PBB} 3$<smiles>CCC(O)COc1ccc(/C=C/Cc2ccc(N)nn2)s1</smiles>
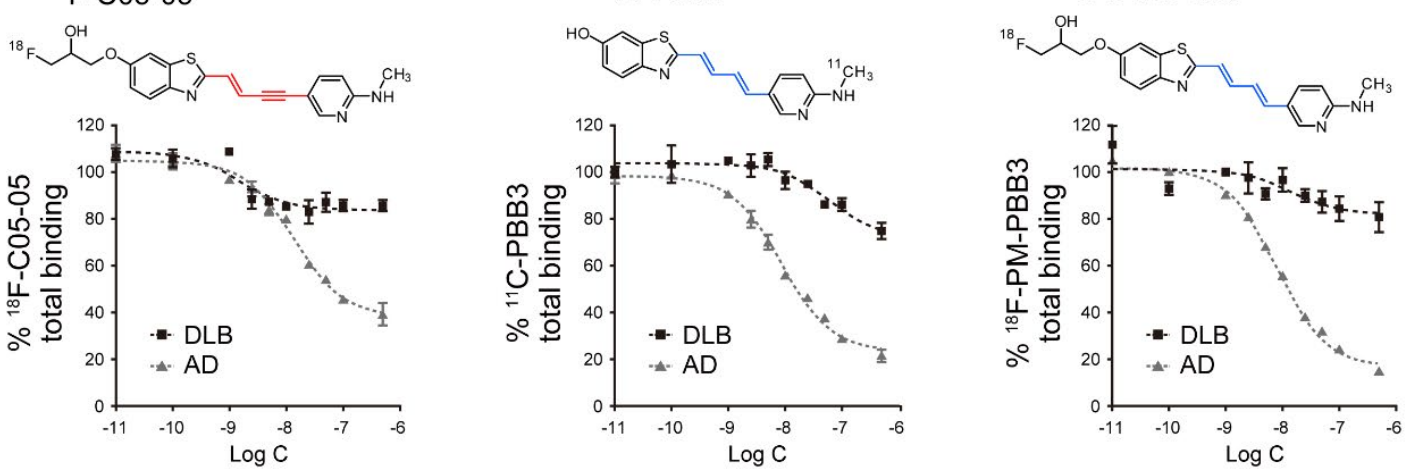

f

\begin{tabular}{lccc}
\hline IC50 $(n M)$ & C05-05 & PBB3 & PM-PBB3 \\
\hline DLB & 1.5 & 58.8 & 26.5 \\
Amygdala & $\left(R^{2}=0.810\right)$ & $\left(R^{2}=0.854\right)$ & $\left(R^{2}=0.854\right)$ \\
AD & 12.9 & 8.6 & 8.0 \\
Frontal cortex & $\left(R^{2}=0.980\right)$ & $\left(R^{2}=0.989\right)$ & $\left(R^{2}=0.994\right)$ \\
\hline
\end{tabular}

Fig. $7 \mid{ }^{18} \mathrm{~F}-\mathrm{C} 05-05$ displays high-affinity binding to $\alpha$-synuclein pathologies in DLB and MSA brain tissues. 
(a and b) Autoradiographic labeling of sections, including the basal ganglia derived from patients with MSA (MSA-1, 2, and 3, also see Supplementary Table 1), amygdala derived from patients with DLB, and substantia nigra derived from patients with PDD (also see Supplementary Table 1), with $10 \mathrm{nM}$ of ${ }^{18} \mathrm{~F}-\mathrm{C} 05-05$ in the absence (a, left) and presence (a, right) of $10 \mu \mathrm{M}$ of non-radiolabeled C0505 , and high-power photomicrographs showing triple fluorescence staining of the section used for ${ }^{18} \mathrm{~F}-\mathrm{C} 05-05$ autoradiography with $30 \mu \mathrm{M}$ of C05-05, LB509, and pS199/202 (b). Areas in b correspond to locations indicated by arrowheads in $\mathbf{a}$. No overt specific binding of ${ }^{18} \mathrm{~F}-\mathrm{C} 05-05$ was detected in the striatopallidal fibers (1) of MSA-1 with mild pathology, weak but clearly noticeable radioligand binding to these fibers (3) was seen in MSA-2 with moderate pathology, and strong radioligand binding to the same subregion (5) was observed in MSA-3 with severe pathology. No significant binding of ${ }^{18} \mathrm{~F}-\mathrm{C} 05-05$ was shown in the areas devoid of a-synuclein pathologies in MSA cases $(\mathbf{2}, \mathbf{4}$, and $\mathbf{6})$. In the amygdala of a DLB case and the substantia nigra of a PDD case, binding of ${ }^{18} \mathrm{~F}-\mathrm{C} 05-05$ was seen in an area harboring abundant Lewy bodies and Lewy neurites (7 and 9). In contrast, no significant binding of ${ }^{18} \mathrm{~F}-\mathrm{C} 05-05$ was noted in an area with a very small amount of a-synuclein pathologies (8 and 10). Immunohistochemistry with pS199/202 indicated the absence of tau deposits in these regions. Scale bars, 1 $\mathrm{mm}(\mathbf{a})$ or $100 \mu \mathrm{m}$ (b). (c-e) Total (specific + non-specific) binding of ${ }^{18} \mathrm{~F}-\mathrm{C} 05-05$ (c), ${ }^{11} \mathrm{C}-\mathrm{PBB} 3$ (d), and ${ }^{18} \mathrm{~F}-\mathrm{PM}-\mathrm{PBB} 3$ (e) in the DLB amygdala (black squares, also see Supplementary Table 1) and AD frontal cortex (grey triangles, AD-2, also see Supplementary Table 1) samples homologously blocked by non-radiolabeled C05-05, PBB3, and PM-PBB3, respectively, with varying concentrations. Data are mean \pm SD in four samples and are expressed as \% of average total binding. (f) Homologous blockades of ${ }^{18} \mathrm{~F}-\mathrm{C} 05-05,{ }^{11} \mathrm{C}-\mathrm{PBB} 3$, and ${ }^{18} \mathrm{~F}-\mathrm{PM}-\mathrm{PBB} 3$ binding described by a one-site model and parameters resulting from curve fits. 